\title{
In vivo bone strain in the mandibular corpus of Sapajus during a range of oral food processing behaviors.
}

Callum F. Ross ${ }^{a}$, Jose Iriarte-Diaz ${ }^{b}$, David A. Reed ${ }^{b}$, Thomas A. Stewart ${ }^{c}$, Andrea B. Taylor ${ }^{d}$

${ }^{a}$ Department of Organismal Biology \& Anatomy

The University of Chicago

${ }^{b}$ Department of Oral Biology

College of Dentistry

University of Illinois at Chicago

'Department of Organismal Biology \& Anatomy

The University of Chicago

${ }^{d}$ Department of Community and Family Medicine

Duke University

Corresponding author:

E-mail address: rossc@uchicago.edu (C.F. Ross)

Keywords: Cebus, Capuchin monkeys, Mastication, Ingestion, Mandibular biomechanics, Feeding ecology 


\begin{abstract}
It has been hypothesized that mandibular corpus morphology of primates is related to the material properties of the foods that they chew. However, chewing foods with different material properties is accompanied by low levels of variation in mandibular strain patterns in macaques. We hypothesized that if variation in primate mandible form reflects adaptations to feeding on foods with different material and geometric properties, then this variation will be driven primarily by differences in oral food processing behavior rather than differences in chewing per se. To test this hypothesis, we recorded in vivo bone strain data from the lateral and medial surfaces of the mandibular corpus during complete feeding sequences in three adult male Sapajus as they fed on foods with a range of sizes and material properties. We assessed whether variation in mandibular corpus strain regimes is associated with variation in feeding behaviors and/or chewing on different foods, and we quantified the relative variation in mandibular corpus strain regimes associated with chewing on foods of different material properties versus a range of oral food processing behaviors (incisor, premolar, and molar biting; pulling on incisors; mastication). Feeding behavior had a significant effect on mandibular corpus strain regimes, as did chewing side and the cycle number in a feeding sequence. However, food type had weaker effects and usually only through interaction effects with chewing side and/or cycle type. Strain regimes varied most across different chew sides, then across different behaviors, and lastly between mastication cycles on different foods. Strain magnitudes associated with premolar, molar, and incisor biting were larger than those recorded during mastication. These data suggest that intra- and inter-specific variation in mandible morphology is a trade-off between performance requirements of different oral food processing behaviors and of variation in chewing side, with direct effects of food type being less important.
\end{abstract}




\section{Introduction}

The relationship between diet and mandible morphology in primates is poorly understood. Dietary category (e.g., folivory, frugivory) and food material properties (FMPs; e.g., toughness, Young's modulus) have been hypothesized to be functionally related to the shape of the primate mandibular corpus (Beecher, 1977; Beecher, 1979; Bouvier, 1986a, b; Hylander, 1979b, 1988; Ravosa, 1991a, 2000; Wallisch et al., 2009a, b). However, these relationships are not consistent across primate clades, suggesting that it is not just dietary category and FMPs that drive form-function relationships in primate mandibles (Ross et al., 2012; Ross and IriarteDiaz, 2014). Primates engage in a wide range of feeding and non-feeding behaviors that place varying demands on the mandible (Daegling, 1992, 1993, 2002, 2007; Daegling and Grine, 2006; Daegling and McGraw, 2007; Hylander, 2013; Ross and Iriarte-Diaz, 2014; Ross et al., 2012; Smith, 1984; Terhune et al., 2011)). These include pre-ingestive and ingestive food processing behaviors such as gouging, stripping, peeling, husking, incisor biting, premolar biting, and molar biting, as well as intra-oral food transport, food manipulation, mastication, and swallowing. Important non-food processing behaviors employing the mandible include yawning, licking, drinking, grooming, agonistic threat displays, and vocal communication.

The relative importance of performance of these different behaviors for mandibular form is unknown. Some behaviors such as vocalization, yawning, licking, threat displays, and drinking elicit low bone strain magnitudes in the mandible and are therefore assumed to be less important determinants of aspects of primate mandibular form related to its mechanical strength than is feeding (Hylander, 1979c, 1984)although for a contrasting opinion see (Daegling, 2012). In contrast, bone strain magnitudes in the mandibular corpus and symphysis recorded when animals bite on a force transducer with their incisors, premolars, and molars can be relatively high, and often higher than those recorded during mastication (Hylander, 1979c, 1984; Ross and Metzger, 2004). This suggests that an evaluation of bone strain regimes in the mandible during a broad range of feeding behaviors might be informative about the determinants of mandibular form. The phrase "feeding behavior" has been used to refer to a wide range of behaviors, from general behaviors such as grazing, browsing, "high selectivity," and "low selectivity" (Van Soest, 1996) to specific gape cycle types, such as chewing, gnawing, 
and swallowing (Hiiemae, 2000) 244; see Ross and Iriarte-Diaz [2014] for a recent review of the hierarchical levels of feeding behavior terminology in primate feeding biomechanics and ecology.) In this study, we focus on a range of behaviors that we define as oral food processing behaviors, including incisor, premolar, and molar biting, pulling on incisors, and mastication, behaviors during which mechanical forces are applied to food items through the mandible, accompanied by jaw muscle forces and reaction forces acting on the mandible from the food item, teeth, and jaw joints (e.g., Hiiemae, 1976, 1978).

Is variation in mandibular corpus morphology related to variation in what primates masticate/chew?

One possibility is that interspecific variation in mandibular shape, as estimated by external dimensions of the mandibular corpus, does not reflect adaptation to variation in the nature of mandibular stress, strain, and deformation when chewing different foods (see Table 1 for definitions), but adaptation to variation in the amount of strain (some integral of strain magnitude and/or number of cycles) associated with chewing. For example, Hylander (1979b) suggested that mastication might influence mandible morphology through selection to improve mandibular resistance to bone fatigue. Noting that the mandibular corpora of colobines are deeper than those of related cercopithecines, that most colobines are highly folivorous, and that leaves require more masticatory cycles than fruit, Hylander (1979b: 230) hypothesized that the relatively deeper mandibular corpora of colobines might function to "prevent mandibular bone fatigue due to cyclical repetitious bending loads." This hypothesis then was applied to explain patterns of mandibular variation between grass-eating cercopithecines (gelada baboons) and frugivorous cercopithecines (Hylander 1979b), across living and fossil prosimians (Ravosa, 1991b, 1992, 2007), an extensive sample of cercopithecoids (Ravosa, 1996), and apes (Ravosa, 2000; Taylor, 2002, 2006a, b).

Another possibility is that interspecific differences in mandible morphology reflect differences in the nature of mandibular loading, stress, and strain regimes associated with mastication of different foods. In macaques, mastication of different foods (apple, dried peach, celery) elicited different orientations of principal strains $\left(\varepsilon_{1}, \varepsilon_{2}\right.$; different strain regimes) on the 
lateral aspect of the balancing side mandibular corpus (Fig. 1; Hylander, 1979c: Table 10). Moreover, mastication on different foods elicits differences in relative amplitude and timing of activation of jaw elevator muscles (Hylander and Johnson, 1994). The jaw elevator muscles apply large external forces to the mandible during the power stroke of mastication, so variation in their activity associated with mastication of different foods might be associated with variation in mandibular strain, stress, and deformation regimes. Moreover, because the orientation of the bite reaction force acting on the mandible is a function of both dental occlusal morphology and the direction of jaw movement during occlusion (Hildebrand, 1931; Beyron, 1964; Ingervall, 1972; Suit et al., 1976; Woda et al., 1979; Ekfeldt and Karlsson, 1996; Rilo et al., 2009), the variation in occlusal morphology between different primates might result in different bite force orientations during the power stroke of mastication, which in turn could result in, for example, different twisting moments acting on the corpus (Hylander, 1979b, 1988).

Thus, theoretical considerations as well as in vivo strain and comparative morphometric data can be invoked in support of the hypothesis that variation in primate mandibular corpus morphology reflects adaptation to variation in the amplitude and nature of strain associated with mastication of foods with different material properties. However, there are reasons for thinking that the influences on external mandible morphology of masticating foods of different material properties might be relatively minor. Although food-related differences in principal strain orientations on the lateral aspect of the corpus during mastication reported by Hylander (1979c: Table 10) are statistically significant, the differences are very small ( 2 degrees; Fig. 1), raising questions about their biological significance. Consider, for example, that the effects of chewing side on principal strain orientations are at least as large as, and usually larger than, effects of food type (average chew side difference $=26^{\circ}$ in Table 8 of (Hylander, 1979c; Hylander et al., 1987). If large differences in strain regimes associated with chewing side demand trade-offs in mandibular corpus design, these might swamp effects on mandible morphology of smaller differences in strain regimes associated with chewing foods with different material properties. 
Another issue, particularly relevant to this paper, is the fact that the majority of the variance in both the relative timing of jaw muscle EMGs and jaw kinematics is found between gape cycles within feeding sequences, not between mastication sequences on different foods (Iriarte-Diaz et al., 2011; Vinyard et al., 2008). A feeding sequence begins when a food item is ingested and ends with the final swallow of that item. In experimental settings, when only one food item is ingested at a time, a large source of between-sequence variation in jaw muscle EMG activity and jaw kinematics is probably the different foods, which in the studies cited above included dried fruits, nuts, bamboo, leaves, and gelatin candies. However, there is also within-sequence variation in jaw muscle EMG and kinematics related to, and probably elicited by, variation in food bolus size and material properties resulting from intra-oral processing of the ingested food item, including food transport, fragmentation, and mixing with saliva (Hiiemae, 2000). The key point for the current paper is that jaw kinematics and jaw muscle EMG relative timing are important determinants of the loading, stress, strain, and deformation regimes in the mandibular corpus during feeding. Consequently, if jaw kinematics and EMG relative timing vary more within than between sequences, then mandibular corpus strain regimes probably also vary more within than between sequences. If mandibular corpus design has to accommodate not only variation in stress and strain regimes associated with chewing side, but also within-sequence variation associated with food processing, effects on mandibular corpus morphology of inter-sequence variation in the properties of foods being masticated may be insignificant or undetectable.

Is variation in mandibular corpus morphology related to variation in oral food processing behavior?

Primate mandibular corpus morphology has also been argued to vary between species that commonly employ different oral food processing behaviors. For example, Hylander (1979a) noted that mangabeys (Lophocebus albigena) have relatively deeper mandibular corpora than cercopithecines that spend less time using their incisors to bite hard fruits. He hypothesized that the relatively deeper mandibular corpora of mangabeys might have evolved to resist high sagittal bending moments associated with frequent incisor biting (Hylander, 1979a). Strain in 
the neck of the mandible varies between incision and mastication (Hylander, 1979a; Hylander and Bays, 1979), strain orientations on the labial surface of the symphysis vary widely with oral food processing behavior (Hylander, 1984), and strain orientations on the lateral aspect of the corpus during ingestion incision and isometric biting frequently differ from those recorded during mastication (Hylander, 1979c)Fig. 1). Thus, not only do mandibular corpus stress, strain, and deformation regimes differ significantly across oral food processing behaviors, but this variation appears larger than that experienced during mastication on different foods. If this is indeed the case, it suggests that inter-specific variation in mandibular corpus morphology might more directly reflect variation in the daily frequency and importance of different oral food processing behaviors (such as incisor biting versus premolar biting versus molar biting/mastication), rather than variation in the material properties of foods being chewed.

The relationships between variation in mandibular corpus morphology and variation in feeding behavior have been explored in a number of morphometric studies, with mixed results. Behavioral differences are argued to underlie differences in mandible shapes between gouging and non-gouging close-relatives (Vinyard et al., 2003; Vinyard and Ryan, 2006). Common marmosets (Callithrix jacchus) do not generate relatively high bite forces during tree gouging, predicting no significant differences in mandibular corpus morphology compared with Saguinus and Saimiri, a prediction confirmed by Vinyard and Ryan (2006). However, gouging primates do generate gouging forces at relatively wider jaw gapes, which in Callithrix jacchus is facilitated by lower condyle heights and longer jaw-muscle fiber lengths, a musculoskeletal configuration that allows common marmosets to operate over a more favorable portion of the length-tension curve at larger gapes (Vinyard et al., 2003; Eng et al., 2009; Taylor et al., 2009). In this case, inter-specific variance in feeding behavior (gape) exerts a strong influence on mandible morphology.

Daegling and McGraw (2001) compared mandibular corpus morphology in sympatric and similar-sized Colobus polykomos and Procolobus badius. C. polykomos not only consumes more seeds than $P$. badius, but it also harvests seeds from "thick woody pods of Pentaclethera macrophylla" and was predicted to have absolutely and relatively larger mandibular corpora (Daegling and McGraw, 2001). Not only were no differences found in symphyseal robusticity 
and corporal breadth at the $\mathrm{M}_{2}$, but corpus height only distinguishes $C$. polykomos males from P. badius males; C polykomos females are not distinct in corpus height. In a later study, Daegling and McGraw (2007) noted that Lophocebus employs incisor biting more frequently during hard object feeding than sympatric Cercocebus, predicting that Lophocebus would have a deeper symphysis and postcanine corpus and that Cercocebus would have a labiolingually thicker and larger symphysis and a larger postcanine corpus area to resist shearing forces. Lophocebus does have a deeper postcanine corpus, as predicted, but in contrast with predictions, the two species do not differ significantly in corpus cross-sectional area (at $\left.\mathrm{M}_{2}\right)$, Lophocebus does not have a deeper symphysis, and the symphysis of Cercocebus is stronger in coronal bending than that of Lophocebus (Daegling and McGraw, 2007).

Taylor (2006b) compared the load resistance abilities of mandibles of Pongo species from Borneo-Pongo pygmaeus wurmbii and Pongo pygmaeus morio-with those of Sumatran Pongo-Pongo pygmaeus abelii. Both species of Bornean orangutans eat relatively tougher foods than $P$. p. abelii, using a range of behaviors, including powerful incision during ingestion and processing. Relative to abelii, both morio and wurmbii have deeper mandibular corpora and larger mandibular condyles relative to their incisor load arms, increasing their resistance to parasagittal bending and condylar compression during incision, but neither of the Bornean species has the broader mandibular corpus relative to abelii predicted for resisting torsional stresses generated during incision, and only morio has the deeper symphyseal shape predicted for resisting coronal bending of the symphysis during incision (Taylor, 2006b; Vogel et al., 2014). The significance of these results is constrained by the lack of detailed data on differences in feeding behaviors between the different species and subspecies of orangutans. However, it is noteworthy that deeper mandibular corpora relative to incisor load arms characterize both the hard object feeding orangutans and Lophocebus. This suggests that variation in the degree of powerful incisor biting might be a determinant of variation in mandibular corpus morphology.

The relationships between dietary toughness, feeding behavior, and mandible corpus morphology among Cebus/Sapajus species, the species studied for this paper, are also not clear-cut. Sapajus sp. are more durophagous than Cebus capucinus, employing their jaws in a range of extra-oral food processing and extraction behaviors. As predicted, Sapajus apella has 
improved jaw-muscle leverage (e.g., Cole, 1992; Wright, 2005, 2009), and female S. apella have relatively more robust mandibular corpora than female $C$. capucinus, with more cortical bone in the cross-section (Daegling, 1992). However, male apella do not differ significantly from male capucinus, and although Sapajus libidinosus process and consume foods of exceptionally high toughness compared to Cebus olivaceus and S. apella, S. libidinosus' mandibular corpus shape does not significantly different from that of S. apella (Wright, 2005, 2009).

In this paper, we explore the relationships between feeding behaviors and patterns of strain in the mandibular corpus of Sapajus capuchin monkeys to better understand how mandible shape might be related to deformation and strain regimes during different behaviors. To evaluate the extent to which variation in food processing behavior is related to variation in mandibular strain regimes, we collected in vivo bone strain data from the mandibular corpora of three robust tufted capuchins (Sapajus sp.; (Lynch-Alfaro et al., 2012; Wright et al., 2015) while they performed a wide range of food processing behaviors. The specific aims of this study were to test the following hypotheses: 1) variation in oral food processing behaviors, such as incisive and premolar biting and pulling, is associated with significant variation in strain regimes in the mandibular corpus, 2) mastication of foods with different material properties results in significant variation in strain regimes in the mandibular corpus, and 3) variation in strain regimes associated with different oral food processing behaviors is greater than variation in strain produced by mastication on foods of different material properties. The mandibular corpus was selected for study because it has been the focus of much of the prior work on mandibular biomechanics (Bouvier, 1986a, b; Daegling, 1989; Daegling, 1990; Daegling, 2007; Hylander, 1979b, 1988; Ravosa, 1991a, 2000; Taylor, 2002, 2006b; Wallisch et al., 2009a, b), because it is commonly preserved in the fossil record, and because it is easily accessed in vivo.

\section{Materials and Methods}

\section{Subjects}

All procedures were approved by the University of Chicago IACUC. Three adult male Sapajus sp. served as subjects. The three individuals were robust, tufted capuchin monkeys, and can therefore be assigned to the genus Sapajus following Lynch-Alfaro (2012) and Wright et 
al. (2015). ${ }^{1}$ The subjects had all of their teeth and displayed no obvious asymmetries, diseases, or deformities of their feeding systems. At least three months prior to data recording, four Vitallium ${ }^{\mathrm{TM}}$ bone screws were implanted in their mandibles and four in their zygomatic arches. These percutaneous screws served as anchoring points for reflective markers used for recording of jaw kinematic data reported elsewhere (Iriarte-Diaz et al., 2011; Reed and Ross, 2010; Ross et al., 2012). In this study, kinematic data for two individuals were of high enough quality to be used to evaluate the effects of surgical strain gage placement on feeding behavior. It was not possible to rigorously compare jaw kinematics before and after screw placement. However, in the several months of data collection after placement of screws, the animals appeared to feed normally, including vigorous ingestion and mastication of a wide range of food items. They displayed no preferences for chewing side and their body weights were not affected by screw placement.

\section{Strain-gage placement}

During one experiment on each animal, three delta rosette strain gages (SA-06-030WY120, Micromeasurements, Raleigh, NC) wired in a three-wire quarter-bridge circuit were attached to the mandibular corpus below $\mathrm{M}_{2}-\mathrm{M}_{3}$. Two gages were placed on the lateral surface and one gage on the medial surface (Fig. 2). The animals were food-deprived for 24 hours before each experiment and then sedated with ketamine $(4 \mathrm{mg} / \mathrm{kg})$ and dexmedetomidine (150-200 $\mu \mathrm{g} / \mathrm{kg}$ ) prior to anesthesia with inhalant isofluorane delivered in $\mathrm{O}_{2}$ (Theriault et al., 2008). After an adequate plane of anesthesia was achieved, a small incision was made in the skin overlying the inferior border of the corpus. Access to the lateral surface of the corpus required no disturbance of the masseter or buccinator muscles. The anterior digastric muscle in Sapajus has a large fleshy attachment to the medial surface of the corpus, extending posteriorly from the symphysis to the medial pterygoid attachment area and from the inferior edge of the corpus to the mylohyoid line. Consequently, access to the medial corpus gage site required detachment of a small portion of the anterior digastric from the corpus. As noted below, these

\footnotetext{
${ }^{1}$ We do not have definitive DNA evidence or geographic locality of the individuals' ancestral populations and there is considerable within-species diversity in Sapajus. Thus, we do not assign the individuals to species.
} 
procedures did not significantly impact jaw kinematics in the two animals in which this could be evaluated.

At each gage site, the periosteum was elevated to expose the cortical bone, a small area of the bone was degreased with clinical grade chloroform, and then the rosette was bonded to the bone with a cyanoacrylate adhesive. To prevent movements of the lead wires from causing strain in the gage circuit, the wires were bonded to the bone for 3-4 mm using the same adhesive. Following bonding of the strain gage and wires, the incision was sutured closed with the lead wires of the strain uge passing out through the incision. The wires were secured to the skin in areas where skin movement is minimal. Indwelling electromyography (EMG) electrodes were implanted in the anterior and posterior temporalis, superficial and deep masseter, and medial pterygoid muscles. Radiographs were taken to document strain gage position and orientation.

The animals were placed in a long-sleeved jacket and then secured in a commercially available restraint (XPL-517-CM, Plas Labs, Lansing, MI) that limited trunk movements while enabling the head and neck to move freely. The jacket sleeves were secured through pulleys to friction blocks that enabled the arms to be released to allow the animal to feed itself. The animals were allowed to recover for at least one hour after isofluorane anesthesia and at least two hours after ketamine sedation before data collection. Each of the three elements of the rosettes was connected to form one arm of a Wheatstone Bridge, with excitation at two volts. Voltage changes were conditioned and amplified on a Vishay $2310 \mathrm{~A}$ system and then recorded using Vicon Motion analysis software. The animals were presented with a range of foods and bone strain, EMG, and jaw kinematic data were recorded while the animals ingested and chewed these foods. After each recording session (= Experiment), the animal was again anesthetized, the gages were removed, the wound cleaned and closed with sutures, analgesics and antibiotics were administered, and the animal was returned to its cage. All animals recovered from all surgeries and recordings without complications.

\section{Foods sampled}

The animals were presented a variety of foods ranging in material and geometric 
properties in order to elicit the wide range of feeding behaviors observed during prior training sessions. The material properties of some of the foods presented to the animals were available in the literature and are presented in Table 2. These material properties were not used in statistical analyses and are only presented for qualitative comparisons by the reader. Though we lack data on the material properties of all the parts of all of the foods ingested and masticated, the data that we do have show a substantial range of material properties in both Young's modulus (ranging between 8.7-2978 MPa) and toughness (ranging between 106-965 $\mathrm{J} \mathrm{M}^{-2}$; Table 2). Geometric properties (size and shape) were not recorded for the food items; however, the food items were presented whole, including Brazil nuts, almonds, and walnuts in the shells. This represents a range of sizes, from the Brazil nuts and walnuts in shells to almonds. Not all animals ate all food types in all experiments, so Table 2 does not summarize the full range of material properties in all experiments. Moreover, the FMPs available to us (Table 2) are from the flesh on the inside of the nuts, not the shells on the outside.

\section{Kinematic analysis}

Rigid body kinematics of the jaws were calculated in Matlab using scripts from the KineMat toolbox (http://isbweb.org/software/movanal/kinemat/). To describe mandibular movement with respect to the fixed cranium, we used instantaneous helical angles, which are similar to Cardan/Euler angles that describe the rotation of a rigid body from one time step to the next (Woltring et al., 1985). Feeding sequences were divided into discrete feeding cycles (between consecutive maximum gapes). Gape cycles were standardized in two ways: first, timing was standardized by dividing each cycle into 100 time steps (percentages of the gape cycle); second, actual timing was maintained, but cycles were aligned so that maximum gape was centered at zero. For two of the individuals ( $C$ and $S$ ), it was possible to compare average kinematic values recorded prior to these strain gage experiments with values recorded during the experiments themselves.

\section{Oral food processing behavior categories}

Video recordings of the experiments were used to divide gape cycles into the following 
categories: incisor, canine, premolar, and molar ingestion bites; canine and incisor pulls; and mastication cycles. Biting side for incisor and premolar bites was determined from the videos; biting side for the mastication cycles was determined from analysis of the 3D jaw kinematics. Gape cycles in which the right molars were moving medially during the slow-close phase of the gape cycle were assigned to right chews; gape cycles in which the left molars were moving medially during the slow-close phase of the gape cycle were assigned to left chews.

\section{Strain analysis}

The strain data were sampled at a rate of $\geq 1,000 \mathrm{~Hz}$, then converted to microstrain $(\mu \varepsilon$, $1 \times 10^{-6}$ ) using calibration files made during the recording sessions. Sequences were selected for analysis on the basis of the quality of the strain data and were rejected if there was obvious movement artifact. All gape cycles in a sequence were included except those where the magnitude of the strains decreased so as to be unreliably distinguishable from noise (which ranged from 5-10 $\mathrm{mV}$ in each channel).

Strain $(\varepsilon)$, measured in $\mu \varepsilon$, is a dimensionless unit equaling the change in length of an object divided by its original length. The maximum principal strain $\left(\varepsilon_{1}\right)$ is usually the largest tensile strain value, while the minimum principal strain is usually the largest compressive strain value $\left(\varepsilon_{2}\right)$. The maximum shear strain $\left(\gamma\right.$-max) is equal to $\varepsilon_{1}$ minus $\varepsilon_{2}$. Selected feeding sequences were analyzed using custom written software in IGOR Pro (Versions 4.0 and 6.43a). Specifically, the direction of $\varepsilon_{1}$ relative to the A-element of the gage, the magnitude of the shear strains, and the ratio of maximum to minimum strains $\left(\left|\varepsilon_{1} / \varepsilon_{2}\right|\right)$ were calculated with standard equations. The magnitude and timing of the peak shear strains were calculated for each power stroke, then the direction of the maximum principal strain and the ratio of maximum to minimum strains at the same point in time were calculated.

The orientations and magnitudes of $\varepsilon_{1}$ and $\varepsilon_{2}$ strain "vectors" recorded at the time of peak strain were plotted and visualized prior to analysis (Supplementary Online Material [SOM] Figures S1-S3). (Although strain is not strictly speaking a vector, the orientation and magnitude of the principal strains can be visualized as such.) These plots facilitate estimation of relative magnitudes of peak tensile and compressive strains, as well as an appreciation of the range of 
strain orientations recorded and the relationships between strain orientations and strain magnitudes. The strain vector plots were created by converting strain orientations (in degrees) and magnitudes (in $\mu \varepsilon$ ) to polar coordinates. The orientations of the vectors relative to the mandibles were determined from notes and radiographs taken during the surgeries, using the postcanine tooth row as the reference plane. In lateral radiographs, this plane was defined as a line from the most posterior point on the occlusal surface of the lower third molar $\left(M_{3}\right)$ to the paraconid of the first lower premolar $\left(\mathrm{P}_{2}\right)$.

Data from three gages placed around the circumference of the mandible allow calculation of the normal strain distribution across the section of the gages. From this distribution, maximum and minimum normal strains for the cross section and the orientation of the neutral axis within the cross section were then calculated using a custom macro for Igor Pro modified from one provided by B. Demes, Stony Brook University (Demes, 1998; Demes, 2007; Demes et al., 2001; Rybicki et al., 1977)). The orientation of the neutral axis provides one measure of the strain and deformation regimes during feeding. To calculate the orientation of the neutral axis of bending, normal strains (strains normal to the plane of the cross section) were calculated from peak principal strains recorded during each bite. Cross-sectional geometry of the mandible was determined from CT scans ( $1 \mathrm{~mm}$ slice thickness) of the specimens. Normal strains were calculated assuming that material properties are homogeneous through the cross section.

Data analyses were performed in Matlab (R2012B), IBM SPSS Statistics (Version 22), Igor Pro 4.0 (Lake Oswego, OR), and Oriana 2.02e (Kovach Computing Services, www.kovcomp.com). Circular statistics were calculated for the orientations of $\varepsilon_{1}$ at each gage site, with chew cycles grouped according to cycle type (incisor biting, premolar biting, mastication) and biting side (i.e., whether the animal bit ipsilateral or contralateral to the gage site, yielding "working-side" and "balancing-side" chews, respectively). Descriptive statistics presented are: the angle $(\mu)$ of the mean vector relative to the tooth row; the length of the mean vector $(r)$, ranging from 0 to 1 , with a larger $r$ value indicating the observations are clustered more closely around the mean; the concentration ( $\mathrm{k})$, the maximum likelihood estimate of the population concentration (Fisher, 1993; Mardia and Jupp, 2000)a parameter 
specific to the von Mises distribution that measures the departure of the distribution from a uniform distribution, when $\mathrm{k}=0$ the distribution is uniform, with higher values converging on a normal distribution); the circular variance $V=1-r$; the circular standard deviation $S=(-2 \ln (r))^{1 / 2}$ (in radians); the standard error of the mean (formula 4.42 in Fisher, 1993); and the weighted mean vector, which is the mean orientation of $\varepsilon_{1}$ weighted by the magnitude of $\varepsilon_{1}$. Note that the length of the mean vector is not an estimate of the mean magnitude of $\varepsilon_{1}$, but instead an estimate of the degree to which the $\varepsilon_{1}$ orientations cluster. We used Rayleigh's test of uniformity to determine whether all strain orientations are equally likely. A significant Rayleigh's test indicates that strain or neutral axis orientations are significantly non-uniform; i.e., there is a predominant orientation. Watson's $U^{2}$ Test is used to determine whether the data are derived from a von Mises distribution and, hence, whether parametric tests of strain orientations were appropriate.

To test the third hypothesis - that variation in strain regimes is higher across different behaviors than across chews on different foods-at each gage site in each experiment the variation in $\varepsilon_{1}$ orientation was quantified across all non-chew cycle types, all chews (including left and right chews), and for left and right chews separately using $S$, the range of mean $\varepsilon_{1}$ orientation, and the range of weighted mean $\varepsilon_{1}$ orientation. The ranges of means were calculated by computing the differences between the highest and lowest mean values of $\varepsilon_{1}$ orientations across behaviors, across chews on different foods, and within and across chewing sides. These values were then tabulated for comparison. Variation in neutral axis orientation was assessed using $\mathrm{S}$ and the range of mean orientation. (Weighted means cannot be calculated for neutral axis orientation.)

Methods for fully nested hierarchical analyses of variance for angular data are not currently available. Instead, two-way ANOVAs of angular data were performed using the Circular Statistics Toolbox in Matlab 2012b to test for effects of food and behavior on orientations of principal strain and neutral axes of bending. Games and Howell post-hoc tests were performed. Balancing side chews (contralateral to gages) and working side chews (ipsilateral to gages) were tested separately. Because these failed distributional assumptions required for parametric tests, mean $\varepsilon_{1}$ and neutral axis orientations recorded during different 
behaviors and during eating of different foods were compared using a nonparametric MardiaWatson-Wheeler test. The effects of food type and chewing side on mean $\varepsilon_{1}$ magnitude and $\log _{10}\left|\varepsilon_{1} / \varepsilon_{2}\right|$ ratios during chewing cycles were tested using a parametric two-way ANOVA with side as a fixed effect, food type as a random effect, and chew number as a covariate. Grape and date chewing were excluded because the strain magnitudes were too low to be reliable. The effects on $\varepsilon_{1}$ magnitudes of cycle type, food type, and cycle number in the sequence were evaluated using a parametric two-way ANOVA, with cycle type and food as random effects and cycle number as a covariate.

\section{Results}

To evaluate whether gage placement affected feeding kinematics, we compared the jaw kinematics for two subjects ( $C$ and $S$ ) recorded during these strain gage experiments with kinematics recorded in previous experiments when no gages were placed. For experimental subject $C$, we found no clear differences in the mean angular velocity of the mandible (Fig. 3A, B). Kinematic patterns of jaw opening/closing and lateral displacements were qualitatively similar, reaching comparable peak points and points of inflection (i.e., changes in angular velocity) at similar times between data collected with the strain gages ( $n=1318$ gape cycles) and that collected without gages ( $n=828$ gape cycles). For experimental subject $\mathrm{S}$, we also found similar kinematic patterns between experiments when gages where present $(n=747$ gape cycles) and when absent ( $n=1067$ gape cycles; Fig. 3C, D). Slight differences in the timing of peak angular velocity of the mandible around the Z-axis (i.e., lateral mandible rotation) were found between experiments (Fig. 3D), but otherwise the same kinematic pattern is observed.

Strain orientations and magnitudes are presented for Experiment 183 in Figures 4-6, for Experiment 184 in Figures 7-9, and for Experiment 185 in Figure 10. SOM Figures S1-3 present all the strain vectors for $\varepsilon_{1}$ and $\varepsilon_{2}$ recorded during these experiments. The $\varepsilon_{1}$ magnitude data are summarized in Figure 11. The $\varepsilon_{1}$ data for Experiment 183 are presented in Tables 3 and 4, for Experiment 184 in Tables 5 and 6, and for Experiment 185 in Table 7. ANOVA results for strain magnitudes and ratios are given in Tables 8 and 9. Ranges of circular standard deviations, means, and weighted means are given in Table 10. 


\section{Experiment 183}

In Experiment 183, animal $\mathrm{M}$ ate almonds, banana chips, cashews, dates, and hazelnuts, while strain data were recorded from the right corpus. The available data on Young's modulus $(E)$ of these foods range from 3.2-12 MPa and on fracture toughness $(R)$ range from 105-965

$\mathrm{Jm}^{-2}$ (Table 2). Strain orientations during date chews were excluded from the analyses summarized below because the strain magnitudes were so low that they approached the level of noise in each element of the rosette strain gage (ca. 5-10 $\mu \varepsilon$; Table 4).

\section{Oral food processing behaviors}

During oral processing behaviors, all strain orientations were not equally likely (Rayleigh's test, Table 3). Oral food processing behavior had a significant effect on $\varepsilon_{1}$ orientation at all three gage sites, as well as on neutral axis orientation (upper lateral, $\mathrm{F}=69.5$; lower lateral, $\mathrm{F}=147.4$; medial, $\mathrm{F}=17.02$; neutral axis, $\mathrm{F}=18.0$; all $p<0.001$ ). Strain magnitudes varied across oral processing behaviors and were always higher (mean and maximum) at the upper corpus than the lower corpus site (compare values for upper lateral and lower lateral in Table 3). Mean strain magnitudes ranged from 30-866 $\mu \varepsilon$ at the upper lateral corpus site, from 5-172 $\mu \varepsilon$ at the lower lateral corpus site, and from 15-80 $\mu \varepsilon$ at the medial corpus site (Table 3). The highest $\varepsilon_{1}$ magnitude recorded was $2138 \mu \varepsilon$, recorded from the upper lateral gage site during isometric biting on the contralateral molars (Table 3). The highest strain magnitudes at all gage sites were recorded during biting, not chewing.

The effects of cycle type, cycle number, and food type on $\varepsilon_{1}$ magnitude and $\left|\varepsilon_{1} / \varepsilon_{2}\right|$ ratios were evaluated using two-way ANOVAs, with cycle type and food as random effects and cycle number as a covariate (Table 8a). At all gage sites, cycle number had the strongest effect (i.e., highest F-values) on $\varepsilon_{1}$ magnitude, and at both lateral gage sites cycle type had the next strongest effect. Food type only had a significant effect at the medial gage site. At the two lateral gage sites, cycle type $\mathrm{X}$ food interaction effects were significant.

The most consistent effect on $\left|\varepsilon_{1} / \varepsilon_{2}\right|$ ratios across all gage sites was the interaction effect between cycle type and food type: i.e., the way that cycle type impacts $\left|\varepsilon_{1} / \varepsilon_{2}\right|$ ratios 
varies with food type. At the upper lateral gage site, $\left|\varepsilon_{1} / \varepsilon_{2}\right|$ ratios were significantly impacted by cycle type and by the interaction between cycle type and food type, but neither food type nor cycle number was significant. At the lower lateral gage site, $\left|\varepsilon_{1} / \varepsilon_{2}\right|$ ratios were significantly impacted by all main and interaction effects, but food type and interaction effects were the weakest. At the medial gage site, $\left|\varepsilon_{1} / \varepsilon_{2}\right|$ ratios were significantly impacted by cycle number, food type, and cycle type $X$ food interactions, but not cycle type.

\section{Chewing}

During chewing, all strain orientations were not equally likely (Rayleigh's test, Table 4). ANOVA for circular data revealed variable effects of food type on mandibular corpus principal strain and neutral axis orientation during chewing cycles. During right chews, food type had a significant effect on mean $\varepsilon_{1}$ orientation at the medial $(F=41.1, p<0.001)$ and lower lateral gage sites $(F=3.03, p=0.03)$, but not at the upper lateral gage site. Food type affected neutral axis orientation during right chews $(F=3.5, p=0.02)$. During left chews, food type had a significant effect on mean $\varepsilon_{1}$ orientation at the medial $(F=8.2, p<0.001)$ and upper lateral gage sites $(F=11.5, p<0.001)$, but not at the lower lateral gage site. Food type also affected neutral axis orientation during left chews $(F=9.5, p<0.001)$.

Strain magnitudes varied during chewing on different foods and were always higher at the upper corpus (compare upper lateral and lower lateral strain data in Table 4) and usually higher during chewing ipsilateral to the gage (i.e., on the right side). The lowest mean strain magnitudes were associated with chewing dates $(18 \mu \varepsilon)$, and the highest mean strain magnitudes were associated with chewing on banana chips (128 $\mu \varepsilon$; Table 4). Maximum strain magnitudes ranged between $46 \mu \varepsilon$ (dates) and $1065 \mu \varepsilon$ (banana chips).

To evaluate the effects of food type and chew side during chew cycles on $\varepsilon_{1}$ magnitude and $\left|\varepsilon_{1} / \varepsilon_{2}\right|$ ratios, we used two-way ANOVAs with food as a random effect, chew side as a fixed effect, and cycle number as a covariate (Table $8 b$ ). During chewing cycles, $\varepsilon_{1}$ magnitudes were significantly affected by cycle number at all gage sites and by chew side at the lateral gage sites. Food type did not have a significant effect on $\varepsilon_{1}$ magnitude at any gage site, but there were significant interaction effects between chew side and food type. The most consistent effect on 
$\left|\varepsilon_{1} / \varepsilon_{2}\right|$ ratios across all gage sites was the interaction effect between chew side and food type: the way that food type impacts $\left|\varepsilon_{1} / \varepsilon_{2}\right|$ ratios varies with chew side. $\left|\varepsilon_{1} / \varepsilon_{2}\right|$ ratios are not affected by food type during chewing at lateral gage sites, but they are at medial gage sites.

\section{Chewing versus non-chewing oral behaviors}

Table 10 compares S (circular standard deviation) and ranges of means and weighted means of $\varepsilon_{1}$ orientations across all non-chewing oral food processing behaviors, across all chews, and across left and right chews separately. In Experiment 183, at the upper lateral gage site, $\mathrm{S}$ in $\varepsilon_{1}$ orientations was highest across all chew cycles (working and balancing sides pooled) and next highest across all non-chewing behaviors; at the lower lateral gage site, $\mathrm{S}$ was highest across all non-chew cycles and next highest across all chews; at the medial gage site, $\varepsilon_{1}$ orientation $\mathrm{S}$ was highest across all chews because of the wide range across contralateral chews (i.e., on the balancing side) and the next highest range was across ipsilateral chews; and in the neutral axis orientation, $\mathrm{S}$ was highest on the balancing side and next highest across all nonchew cycles. Comparing ranges of means of $\varepsilon_{1}$ orientations reveals that at the lateral gage sites, the highest ranges are seen across all chews, whereas the second highest ranges are seen across all non-chew behaviors, while the reverse is true at the medial gage site and for the neutral axis. When ranges of weighted mean $\varepsilon_{1}$ orientations are compared in Experiment 183, the widest ranges are seen across all chews, whereas the second widest are seen across all nonchew behaviors.

\section{Experiment 184}

In this experiment, the animal ate walnuts, Brazil nuts, pecans, hazel nuts, almonds, popcorn, mango, apricot, and coconut, while strain data were recorded from the right corpus. The available data on values of $E$ for these foods range from 0.99-325 MPa and values of $R$ range from $105-2979 \mathrm{Jm}^{-2}$.

Oral food processing behaviors 
During oral processing behaviors, all strain orientations were not equally likely (Rayleigh's test, Table 5). Oral food processing behavior had a significant effect on $\varepsilon_{1}$ orientation at all gage sites, but not on neutral axis orientation (upper lateral, $F=104.6$; lower lateral, $\mathrm{F}=40.8 ;$ medial, $\mathrm{F}=12.1 ;$ all $p<0.001$ ).

Strain magnitudes varied across oral processing behaviors and were always higher (both mean and maximum) at the lower lateral gage site (Table 5). Mean strain magnitudes ranged between $77-526 \mu \varepsilon$ at the upper lateral corpus, 98-1273 $\mu \varepsilon$ at the lower lateral corpus, and 96-603 $\mu \varepsilon$ at the medial corpus gage site. The highest mean and maximum strains were always associated with biting on the working-side "premolars/molars."

The effects on $\varepsilon_{1}$ magnitude and $\left|\varepsilon_{1} / \varepsilon_{2}\right|$ ratios of cycle type, cycle number in the sequence, and food type were evaluated using two-way ANOVAs (cycle type and food, random effects), with cycle number as a covariate (Table 8a). As in Experiment 183, in Experiment 184 cycle number had the strongest (or nearly the strongest) effect on $\varepsilon_{1}$ magnitude at all gage sites. Cycle type had the next strongest effect at all gage sites. Food type had a significant effect at all gage sites in Experiment 184, but in all cases the F-values are relatively low. Cycle type $X$ food interaction effects were significant at all gage sites.

As in Experiment 183, the most consistent effect on $\left|\varepsilon_{1} / \varepsilon_{2}\right|$ ratios across all gage sites in Experiment 184 was the interaction effect between cycle type and food type: the way that cycle type impacts $\left|\varepsilon_{1} / \varepsilon_{2}\right|$ ratios varies with food type. At the lower lateral gage site, only cycle number and cycle type $X$ food interactions had significant effects on $\left|\varepsilon_{1} / \varepsilon_{2}\right|$ ratios. At the medial gage site, cycle number and cycle type $X$ food interactions had significant effects on $\left|\varepsilon_{1} / \varepsilon_{2}\right|$ ratios: food type was not significant.

\section{Chewing}

During chewing, all strain orientations were not equally likely (Rayleigh's test, Table 6). During left chews (gages on balancing side), food type had a significant effect on mean $\varepsilon_{1}$ orientation at the medial $(\mathrm{F}=4.11 ; p<0.001)$ and upper lateral gage sites $(\mathrm{F}=11.3 ; p<0.001)$, but not at the lower lateral gage site, nor on neutral axis orientation. During right chews (gages on working side), food type did not have a significant effect on mean $\varepsilon_{1}$ orientation at any of 
the corpus gage sites. A parametric two-way ANOVA of $\log _{10}\left|\varepsilon_{1} / \varepsilon_{2}\right|$ ratios revealed that at the upper gage site both chew side and food type were significant $(\mathrm{F}=16.1 p<0.001 ; \mathrm{F}=8.0, p=$ 0.004 , respectively), but interaction effects were not. At the lower lateral gage site, neither food type nor chew side effects were significant. At the medial gage site, interaction effects $(F=$ $5.8, p<0.001)$ and chew side $(F=7.7, p=0.02)$ were significant, but food type was not.

Strain magnitudes varied with FMP and foods being chewed, with mean strain magnitudes ranging between $41 \mu \varepsilon$ (walnut, upper lateral corpus, left chew side) and $419 \mu \varepsilon$ (almond, lower lateral corpus, right chew side), with maximum strains are as high as $715 \mu \varepsilon$ (Brazil nut, lower lateral corpus, right chews). The highest maximum strains occur during popcorn chewing at the upper lateral corpus (both right and left chews), the lower lateral corpus (left chews), and the medial corpus (right and left chews). For most foods, there is considerable overlap in mean strains during chewing (relatively high standard deviations); mean chew strains associated with popcorn, our most mechanically challenging food, stand out as usually exhibiting the least amount of overlap with mean chew strains associated with other foods (Table 6).

The effects on $\varepsilon_{1}$ magnitude and $\left|\varepsilon_{1} / \varepsilon_{2}\right|$ ratios of food type and chew side during chew cycles were assessed using two-way ANOVAs with food as a random effect, chew side as a fixed effect, and cycle number as a covariate (Table 8b). As in Experiment 183, in Experiment 184, $\varepsilon_{1}$ magnitudes at the upper lateral gage site were significantly affected by chew side and cycle number. However, unlike Experiment 183, food type did have a significant effect on $\varepsilon_{1}$ magnitudes at the upper lateral gage site, but chew side $X$ food type interaction effects were not significant. At the lower lateral gage site, chew side, cycle number, and chew side $x$ cycle number interactions had significant effects on $\varepsilon_{1}$ magnitudes. Food type was not significant. At the medial gage site, chew side, cycle number, and food type had significant effects on $\varepsilon_{1}$ magnitudes, but interaction effects between chew side and food type were not significant. As in Experiment 183, the most consistent effect on $\left|\varepsilon_{1} / \varepsilon_{2}\right|$ ratios across all gage sites was the interaction effect between chew side and food type: the way that food type impacts $\left|\varepsilon_{1} / \varepsilon_{2}\right|$ ratios varies with chew side. At all three gage sites, $\left|\varepsilon_{1} / \varepsilon_{2}\right|$ ratios are not affected by food type during chewing and this ratio is also not impacted by chew side at lateral gage sites. 
Chewing versus non-chewing oral behaviors

Table 10 compares $S$ and ranges of means and weighted means of $\varepsilon_{1}$ orientations across all non-chewing oral food processing behaviors, across all chews, and across left and right chews separately. At the upper lateral gage site, $\varepsilon_{1}$ orientation $\mathrm{S}$ was highest across all chew cycles (working and balancing sides pooled) and second highest across all non-chews. At the lower lateral gage site, $\varepsilon_{1}$ orientation $\mathrm{S}$ was highest across all non-chew cycles and second highest across all chews. At the medial gage site, $\varepsilon_{1}$ orientation $\mathrm{S}$ was highest across all chews, because of the high value of $\mathrm{S}$ across contralateral chews (i.e., on the balancing side), and second highest during ipsilateral chews. $\mathrm{S}$ in neutral axis orientation was highest on the balancing side and second highest across all chews. The widest range of means at the lateral gage site was across all chews, driven by the wide range across balancing side chews, and the second highest range was across all non-chew behaviors. At the lower lateral gage site, the widest range was across all non-chew behaviors and the second widest range was across all chews. At the medial gage site and the neutral axis, the widest range in mean orientations was across all chews, with the second largest being across left chews. The widest range of weighted means was across all chews (and balancing side chews) at the two lateral gage sites, with the second highest ranges being across all non-chew behaviors. At the medial gage site, the widest range in weighted means was across all chews and the second highest across all left chews.

\section{Experiment 185}

During this experiment, the animal only chose to eat Brazil nuts, so the effects of food type on $\varepsilon_{1}$ orientation during mastication were not assessed. Strains were recorded from the left corpus.

\section{Oral food processing behaviors and chews}

During oral processing behaviors, all strain orientations were not equally likely (Rayleigh's test, Table 7). ANOVA for circular data using cycle type as a factor revealed cycle type to have a significant effect on $\varepsilon_{1}$ orientation at the medial gage site only $(\mathrm{F}=17.8 ; p<$ 
0.001). One-way ANOVA revealed a significant effect of cycle type on $\log _{10}\left|\varepsilon_{1} / \varepsilon_{2}\right|$ ratios at all three gage sites (upper lateral, $\mathrm{F}=7.1, p<0.001$; lower lateral $\mathrm{F}=15.3, p<0.001$; medial, $\mathrm{F}=$ $9.8, p<0.001)$.

Strain magnitudes vary across oral processing behaviors with average strains ranging between 180-763 $\mu \varepsilon$ (upper lateral corpus), 82-170 $\mu \varepsilon$ (lower lateral corpus), and 118-499 $\mu \varepsilon$ (medial corpus). Strains are always highest at the upper lateral corpus. At medial and lower lateral corpus gage sites, the highest strain magnitudes (both mean and maximum) are associated with premolar biting. At the upper lateral corpus gage site, the highest strain magnitudes were recorded during incisor biting (Table 7).

The range of mean values for $\varepsilon_{1}$ orientation across oral food processing behaviors was $3.1^{\circ}$ at the upper lateral gage site, $5.8^{\circ}$ at the lower lateral gage site, and $41.3^{\circ}$ at the medial gage site (Fig. 10). Mean neutral axis orientation across behaviors only varied by $12^{\circ}$.

\section{Discussion}

The data presented here provide insight into variation in mandibular corpus strain regimes $-\varepsilon_{1}$ orientation and magnitude, the ratio of $\left|\varepsilon_{1} / \varepsilon_{2}\right|$, the orientation of the neutral axis of bending-during a wide range of oral food processing behaviors on a wide range of foods. Although Young's modulus and toughness data are available for some parts of some of these foods (Table 2), material properties data are not available for the intact Brazil nut, walnut, and almond shells that the animals broke open during premolar and molar biting. Thus, the FMP ranges presented in Table 2 might under-estimate the range of FMPs presented to the animals in this study, especially during ingestion cycles when the shells were broken by the animals. Moreover, we do not know whether we have sampled the high-end range of FMPs ingested and chewed by tufted capuchins in the wild (Wright, 2005a, b; Wright et al., 2009; Chalk, 2015). However, these limitations do not impact the analyses performed in this study as FMP data were not included in the statistical analyses.

What do the results presented here mean for the three hypotheses presented in the Introduction? 
Variation in strain regimes due to variation in feeding behavior

The first hypothesis, that variation in oral food processing behaviors elicits significant variation in strain regimes in the mandibular corpus, is corroborated. Variation in oral food processing behavior - represented here by "cycle type," including biting and/or pulling at incisors, premolars, and molars, as well as chewing-had significant effects on $\varepsilon_{1}$ strain magnitudes at all gage sites, in all experiments, except the medial gage in Experiment 183. Moreover, in all experiments at all gage sites, the largest strain magnitudes were recorded during biting, not chewing. When the highest peak strain magnitudes are considered (whiskers in Fig. 11), the behaviors with the highest strains in Experiments 184 and 185 were incisor biting and ipsilateral and contralateral premolar biting, whereas in Experiment 183 the highest peak strain magnitudes at all gage sites were recorded during contralateral molar biting. When highest mean strain magnitudes are considered, ipsilateral molar and premolar biting are associated with the highest magnitudes at all gage sites.

Cycle type variation significantly affected $\left|\varepsilon_{1} / \varepsilon_{2}\right|$ ratios at the upper lateral gage site in Experiment 184, at the two lateral gage sites in Experiment 183, and at all three gage sites in Experiment 185 . Cycle type variation also significantly affected $\varepsilon_{1}$ orientation at seven out of nine gage sites (not at the lateral gage sites in Experiment 185) and the orientation of the neutral axis of bending in Experiment 183, but not 184 or 185. The neutral axis of bending is the axis about which the mandible is bent, so low variation in its orientation suggests that variation in strain orientations and $\left|\varepsilon_{1} / \varepsilon_{2}\right|$ ratios (see below) reflects variation in other deformation regimes, such as twisting and shear.

In addition, in Experiments 183 and 184, the interaction between food and cycle type had significant effects on $\varepsilon_{1}$ magnitude at all gage sites except the medial gage in Experiment 183 , and on $\left|\varepsilon_{1} / \varepsilon_{2}\right|$ ratios at all gage sites. This interaction effect means that the way that food type affects strain magnitudes and principal strain ratios depends on the cycle type, and the way that cycle type affects strain regimes depends on the food being eaten.

Macaque mandibular corpus strain orientations have been shown to vary little across the oral food processing and transducer biting behaviors sampled: chewing, ingestive incisor biting, and isometric transducer biting on incisors and molars (Fig. 1; Hylander, 1979). However, 
the variation across behaviors in the macaque studies is narrower than that documented here. This may be because a narrower range of behaviors was sampled in the macaque studies and/or because their hands were restrained, whereas the capuchins studied here used their hands to feed. It remains to be determined whether macaques would employ as wide a range of feeding behaviors as capuchins under the same experimental conditions, and whether these would result in as wide a range of mandibular loading and strain regimes.

The second hypothesis we evaluated was whether the mastication of foods with different material properties is associated with significant variation in strain regimes in the mandibular corpus. This hypothesis received mixed support. There were only significant effects for food type on strain regimes at the upper lateral and medial gage sites in Experiment 184, and these effects were characterized by low F-values (Table 8b). During chewing cycles, the largest effects on $\varepsilon_{1}$ magnitudes were always cycle number and/or chewing side. Similarly, out of all the six gage sites in Experiments 183 and 184, food type only significantly affected $\left|\varepsilon_{1} / \varepsilon_{2}\right|$ ratios at the medial gage site in Experiment 183 (Table 8b). Again, chew side or cycle number had larger effects on $\left|\varepsilon_{1} / \varepsilon_{2}\right|$ ratios than did food type. Chew side had a significant effect on $\varepsilon_{1}$ orientations at all gage sites during all experiments, except the lateral gage sites in Experiment 185. Controlling for chew side, food type had a significant effect on $\varepsilon_{1}$ orientations at two out of six working side gage sites (i.e., the working side medial and lower lateral gage sites in Experiment 183), at four out of six balancing side gage sites (i.e., the balancing side medial and upper lateral gage sites in both Experiments 183 and 184), and on neutral axis orientation on both working and balancing sides in Experiment 183, but on neither side in Experiment 184. Food/FMP effects on strain regimes during chewing were more common in Experiment 183 than Experiment 184, more common at balancing side than working side gage sites, and more common for $\varepsilon_{1}$ orientations than $\varepsilon_{1}$ magnitudes or $\left|\varepsilon_{1} / \varepsilon_{2}\right|$ ratios. Thus, although chewing different foods was associated with variation in strain regimes, as predicted by Hypothesis 2 , chew side and chew number had larger effects than did food type. Indeed, in Experiments 183 and 184 food type had the smallest (mostly non-significant) effects on $\varepsilon_{1}$ magnitudes and $\left|\varepsilon_{1} / \varepsilon_{2}\right|$ ratios of any of the factors. 
The third hypothesis that we evaluated was whether the variation in strain regimes associated with different oral food processing behaviors is greater than that during chewing on different foods with different material properties. The three metrics used to quantify relative variability were the circular standard deviation $(S)$, the range of mean $\varepsilon_{1}$ orientations, and the range of weighted mean $\varepsilon_{1}$ orientations. The relative variability of strain regimes across different behaviors versus chewing on different foods differs depending on the metric, the individual, and the gage site, nevertheless several patterns can be observed. First, in the majority of the 22 comparisons in Table 10, the widest range or greatest standard deviation in $\varepsilon_{1}$ orientations is across all chews combined, i.e., working and balancing sides combined. This suggests that there is often more variation in strain regimes with chewing side than between different feeding behaviors. Variation in mastication-related strain regimes with chew side is documented for the mandibular corpus of macaques, galagos, and owl monkeys (Hylander, 1979; Hylander et al., 1998) and for the mandibular symphysis of macaques (Hylander, 1984). In macaques, these strain data suggest that the working or biting side mandibular corpus in the molar region is "primarily twisted about its long axis, directly sheared dorsoventrally, and slightly bent in both parasagittal and transverse planes during the power stroke of both mastication and unilateral molar biting" (Hylander, 1988: 56), whereas "the balancing-side mandibular corpus in the molar region is primarily twisted about its long axis, directly sheared dorsoventrally, and powerfully bent in the parasagittal plane during the power stroke of both mastication and unilateral biting" (Hylander, 1988: 56-57). Thus, in macaques, the working side corpus is subjected to relatively greater twisting and the balancing side corpus to relatively greater bending. What are the implications of such working-balancing side differences in deformation regimes for mandibular design in primates? If individual primates spend approximately equal amounts of time chewing on left and right sides, and mandibular corpus morphology reflects adaptations to resist chewing stresses and strains, then mandibular corpus geometry must be a trade-off between resistance to twisting and resistance to parasagittal bending. Exactly how this trade-off would be manifested is difficult to predict, but it does raise the possibility that working/balancing side variation in strain regimes could mask dietary effects on mandibular corpus morphology. 
Second, in the majority of cases in Table 10, variation in chewing related strain regimes is greater on the balancing side than the working side, and in Experiment 184, the highest strain magnitudes at all gage sites were recorded during ipsilateral premolar biting. This might suggest that working side strain regimes have a more consistent and stronger effect on mandibular corpus design than balancing side strain regimes, predicting greater diet-related variation in the degree of resistance to torsional stress than bending stress. Unfortunately, the exact nature of the relationship between torsional stress and mandibular morphology in primates is unclear (Daegling and Hylander, 1991). Moreover, an argument can be marshalled that high bending stresses on the balancing side corpus associated with contralateral chewing and biting might be important in driving mandibular corpus morphology. As summarized in the Introduction, relatively deep mandibular corpora relative to incisor load arms characterize the mandibles of both hard object feeding orangutans and Lophocebus, and the highest strains in Experiment 184were at the upper lateral gage site during contralateral biting. If bending is the predominant loading regime in the balancing side corpus in these animals as in macaques, this might reflect a greater importance of balancing side loading regimes. The significance of this greater variation in balancing side strain regimes is therefore unclear.

Third, if the effects of chew side on variation in strain regimes are ignored, the range of $\varepsilon_{1}$ orientations across different feeding behaviors exceed those recorded during chewing on either side in 10 of 22 cases, and exceed those recorded on at least one side in 19 of 22 cases. These data do not provide strong support for the hypothesis that variation in strain regimes associated with different behaviors always exceeds variation associated with chewing on different foods, but they do suggest that variation in strain regimes associated with different feeding behaviors can exceed that associated with chewing on different foods. Stronger support for the third hypothesis comes from previously published data on macaques (Hylander, 1979c), in which strain orientations recorded from the lateral aspect of the mandibular corpus show less variation during mastication of different foods $\left(<2^{\circ}\right)$ than across different behaviors (Fig. 1; Hylander, 1979c; average difference $=26^{\circ}$ in Table 8). Moreover, published data from non-primate mammals suggest that their mandibles also experience low levels of variation in strain regimes during mastication of different foods. Williams et al. (2011) report no significant 
differences between strain orientations and $\left|\varepsilon_{1} / \varepsilon_{2}\right|$ ratios recorded during rumination chewing and ingestion chewing in goats (Williams et al., 2011). ${ }^{2}$ In rabbits, mean working side $\varepsilon_{2}$ orientations varied by only $1^{\circ}$ across chewing of pellets ( $33^{\circ}$ relative to occlusal plane), hay $\left(34^{\circ}\right)$, and carrot $\left(33^{\circ}\right)$; balancing side $\varepsilon_{2}$ orientations varied more (pellets, $73^{\circ}$; hay, $76^{\circ}$; carrot $\left.92^{\circ}\right)$, while strain orientations during carrot incision were very different $\left(49^{\circ}\right.$; (Weijs and De Jong, 1977). Collectively these data from non-primate mammals suggest that strain regimes in the mammalian mandible often vary little between chews on different foods/FMPs and are more strongly affected by chewing side and, where data are available, chew number.

Finally, it is worth noting that consideration of the standard deviations in Table 10 reveals interesting patterns in variability in strain and neutral axis orientation. In both Experiments 183 and 184, the highest values of $\varepsilon_{1}$ orientation $S$ were found: at the upper lateral gage site across all chew cycles (working and balancing sides pooled), at the lower lateral gage site across all non-chew cycles, and at the medial gage site and neutral axis across contralateral chews. Confirmation of this pattern might suggest differences in relative importance of different behaviors for the morphology of different parts of the mandible.

\section{What do our results reveal about primate mandibular corpus form-function relationships?}

In order for interspecific variation in mandibular (in this case corpus) morphology to be consistently related to interspecific variation in the material or geometric properties of primate foods, three criteria must be met (Ross and Iriarte-Diaz, 2014; Ross et al., 2012)Fig. 12). First, variation in food geometric and/or material properties must elicit variation in feeding behavior that results in variation in mandibular corpus loading regimes (patterns of external forces acting on the corpus) that are in turn associated with variation in mandibular corpus stress and strain regimes. For example, if two foods are of similarly high toughness and Young's modulus, but one (perhaps the larger) is consistently ingested via biting on the premolars while the other is consistently ingested via biting on the molars, then the differences in food geometric properties do elicit different feeding behaviors characterized by different mandibular loading regimes

\footnotetext{
${ }^{2}$ Williams et al. (2011) use the term "ingestion chewing" to refer to chewing of food when it is first ingested; in contrast with "rumination chewing", which refers to chewing of regurgitated food.
} 
(different bite points by definition imply different loading regimes, with or without different jaw-muscle activity patterns). Similarly, if foods with different material properties are chewed in different ways, this might elicit variation in mandibular corpus loading regimes. If these different loading regimes result in different strain regimes then it is possible for natural selection to act on heritable variation in mandibular corpus morphology (under "heritable variation" we include pleiotropic effects of genetic loci (Ehrich et al., 2005; Ehrich et al., 2003), and heritable mechanisms that facilitate plastic responses to variation in strain regimes (Bouvier and Hylander, 1981, 1994; Bouvier and Hylander, 1996a, b; Ravosa et al., 2007a; Ravosa et al., 2007b; Ravosa et al., 2006). This mapping from food material and geometric material properties to strain regimes is shown in Figure $12 \mathrm{~A}$.

This brings us to the second criterion. In order for natural selection to produce changes in mandibular corpus morphology, different mandibular corpus morphologies must perform better under different mandibular corpus stress and strain regimes, and this must improve feeding performance and fitness. For example, if relatively high bending stresses and strains associated with premolar biting are best resisted by increasing relative dorsoventral depth of the corpus, and animals with relatively deeper corpora have improved feeding performance and higher fitness, then natural selection is predicted to associate premolar biting with relatively deeper mandibular corpora. Or, if chewing foods with higher toughness is associated with relatively higher bending stresses, this might also be associated with evolution of increased depth of the mandibular corpus. As shown in Figure 12B, this can be conceived of as a weighting of the importance for mandibular morphology of the range of different oral food processing behaviors and strain regimes.

Finally, if consistent associations between oral food processing behavior, loading regime, strain regime, and corpus morphology are to evolve in different primate lineages, then these selective forces must act in the same morphological and behavioral contexts in those lineages (Kay and Cartmill, 1977; Ross et al., 2002).

The results of the experiments reported here are most germane to evaluating the first criterion discussed above: does variation in food geometric and material properties elicit variation in oral food processing behaviors, including in the way animals chew, resulting in 
significantly different strain regimes in the mandibular corpus? As predicted by our first hypothesis, when the three individuals in our study performed multiple different feeding behaviors using different cycle types, at the majority of sites cycle type had a significant impact on $\varepsilon_{1}$ orientations and magnitudes and on $\left|\varepsilon_{1} / \varepsilon_{2}\right|$ ratios. Food type had either weak or nonsignificant effects on $\varepsilon_{1}$ orientations and magnitudes, and on $\left|\varepsilon_{1} / \varepsilon_{2}\right|$ ratios, except through interaction effects with cycle type (Table 8a). As predicted by the second hypothesis, mastication was also associated with variation in strain regimes in the mandible. However, most variation was nested between cycles within chewing sequences, as reflected in cycle number and, to a lesser extent, across chewing sides. Food type had either weak or nonsignificant effects on strain regimes during chewing, except through chew side by food type interaction effects (Table 8b). Contrary to the third hypothesis, the most consistent effect on variation in $\varepsilon_{1}$ orientation in the mandibular corpus is variation in chewing side, the next most consistent effect is feeding behavior (including biting side): the effects of variation in food material properties on variation in strain orientations are relatively minor. Similar results are seen in Macaca fascicularis, where variation in mandibular corpus strain regimes across behaviors is greater than variation between chews on different foods ipsilateral or contralateral to the strain gages, but there are significant effects of chew side on strain orientations (Hylander, 1979c). These results are not unexpected because variation in the most important determinants of strain regimes in the primate mandible (jaw kinematics and relative timing of EMG activity in jaw elevator muscles) is higher within feeding sequences and across different feeding behaviors than across chews on different foods (Vinyard et al., 2008; Ross et al., 2012; Ross and Iriarte-Diaz, 2014). Together, the results presented here and the data in the literature suggest that if the morphology of the mandibular corpus reflects selection for resistance to patterns of stress and strain experienced during feeding, this morphology must be a trade-off between different behaviors, including chewing side.

It has long been recognized that mandible design reflects trade-offs in performance at a wide range of feeding and non-feeding behaviors (Daegling, 1993, 2007; Daegling and Grine, 2006; Daegling and McGraw, 2007; Hylander, 1979a; Hylander, 1979b, 1984, 2013; Ross and Iriarte-Diaz, 2014; Ross et al., 2012; Taylor et al., 2008; Vinyard and Ryan, 2006; Vinyard et al., 
2003; Vinyard et al., 2009) However, in previous work, the range of feeding behaviors elicited in the laboratory during measurement of bone strain has been rather narrow. The data presented here show that when strains are recorded in animals that can use their hands during feeding, they employ a wide range of oral food processing behaviors and the mandibular corpus experiences a wide range of strain regimes (Fig. 12A). Thus, although we have not sampled the full range of food material properties encountered and processed by Sapajus in the wild (Wright, 2004; Wright, 2005a; Wright, 2005b), we expect that further expansion of this study to a wider range of FMPs will be associated with a greater increase in variation in strain magnitudes and orientations associated with different food processing behaviors compared with those associated with mastication of foods with different properties.

The question of how to evaluate the relative importance for mandible design of these different behaviors and strain regimes brings us to criterion 2 (Fig. 12B). It is very difficult to directly relate variance in primate mandibular corpus morphology to ecologically relevant measures of feeding performance (e.g., short term food or nutritional intake rates), and it is impractical to relate it to fitness. Consequently, the importance of different loading regimes for mandible design has been estimated using data on strain magnitudes and inferences about the daily frequency at which different behaviors occur (Hylander, 1979c). Strain magnitude is important because very high bone strain magnitudes are associated with bone yield and fracture (Burstein et al., 1972; Reilly and Burstein, 1974, 1975), and because strain magnitude is important in triggering adaptive responses of bone remodeling, modeling, and repair (Forwood and Turner, 1995; Frost, 1987, 2000; Turner, 1998). Consequently, behaviors associated with high magnitude strain regimes may be more important determinants of mandibular form than behaviors associated with low strain behaviors. However, daily frequencies of different behaviors are also important because bones can be weakened by fatigue, so that behaviors producing lower-than-maximum strain magnitudes over long periods of time (in the case of tension) or many cycles (in the case of compression) might be as-or more-important influences on mandibular form than high strain magnitude behaviors (Carter et al., 1981; Carter et al., 1976; Zioupos et al., 2001; Zioupos et al., 1996). The relationships between strain magnitudes and effects of fatigue (loading time to yield or failure) in primate feeding systems 
are therefore crucial data to obtain, but are currently unavailable. These relationships have been studied in some human bones and for bones in several nonprimate species, but they vary among species, bones, loading regimes (Carter et al., 1981; Reilly and Currey, 1999; Zioupos et al., 2001), bone material properties (Carter et al., 1976; Currey, 2004), and temperature, so their relevance for the evolution of the primate feeding system is unclear. It is important to note that here and elsewhere (Ross and Iriarte-Diaz, 2014; Ross et al., 2012) we are not arguing that fatigue resistance has no bearing on primate mandible design, only that the nature and importance of its effects are poorly understood. What is clear is that in order to estimate the relative importance of different feeding behaviors, we need data on strain regimes (including magnitudes) for as complete a range of feeding behaviors and as wide a range of food material properties as possible, along with data on how often these behaviors are employed in the wild (Taylor et al., 2008; Vogel et al., 2014).

The results of this study also show that the largest mandibular corpus strain magnitudes were recorded during premolar and incisor bites, rather than chewing. Hylander's (1979c data on mandibular corpus bone strain in Macaca and Otolemur also reveal higher bone strain magnitudes during isometric biting on a bite force transducer with the molars than chewing with the molars, but lower strain magnitudes during transducer biting with the incisors than ingestion incision. These results, combined with evidence that cyclic loading and long loading times can cause fatigue yielding and failure in bone, suggest that it would be valuable to know the relative proportions of time spent in biting and chewing behaviors in wild primates (Brown, 1997; Daegling and Grine, 2006; Ross and Iriarte-Diaz, 2014; Ross et al., 2012; Taylor et al., 2008).

\section{Conclusions}

The primate feeding behavior/feeding ecology literature is well-populated with data on the relative proportions of different items in primate diets and the proportions of time spent feeding on different items. Recent years have seen significant progress on the material and geometric properties of wild primate foods. The results of our study suggest that in order to understand the relationships between these properties and mandibular morphology, we need 
to know how these properties are related to and elicit different oral food processing behaviors. Data are needed on aspects of wild primate feeding behavior that impact the loading, stress and strain regimes, and histories of primate mandibles, such as where on the mandible bite forces are applied, the likely magnitudes of those forces, the gapes at which they are applied, and how many times they perform different behaviors, including number of cycles and total loading time (e.g., McGraw et al., 2011). These data need to be collected across as wide a range of behaviors as possible, including pre-ingestive, extractive feeding behaviors, such as gouging (Vinyard and Ryan, 2006; Vinyard and Schmitt, 2004; Vinyard et al., 2003; Vinyard et al., 2009; Vinyard et al., 2001)and ingestive processing, such as incisor, canine, and molar biting, in addition to chewing. Non-feeding behaviors such as threat displays (Hylander, 2013; Terhune et al., 2015) can impose significant strains on the mandible, but also impose trade-offs on feeding system design that need to be considered. Thus, the relative frequencies and importance of feeding and non-feeding will need to be factored into more complete biomechanical explanations for variation and evolution of primate feeding system form (e.g., (Taylor et al., 2008).

\section{Acknowledgements}

We thank E. Vogel, N. Yamashita, and B. Wright for the opportunity to present this paper in their symposium Food materials testing and its relevance for primate biology, held at the 2013 Annual Meeting of the American Association of Physical Anthropologists, and for their invitation to contribute to this volume. We thank the staff of the ARC at The University of Chicago for their animal care and expertise and the reviewers of the paper for their feedback. This work was funded by NSF HOMINID and Physical Anthropology grants (BCS 0240865; BCS 0504685; BCS 0725126; BCS 0725147; BCS 0962682). 
Figure legends

Figure 1. Diagrams of mean principal strain orientations and magnitudes recorded from the lateral aspect of the mandibular corpus of three Macaca fascicularis during transducer biting and apple eating, as reported by Hylander (1979). Tracings of radiographs and orientations of reference elements are taken from Hylander (1979: Fig. 8). Strain magnitude and orientation data are from Hylander (1979: Tables 3 and 8).

Figure 2. 3D surface renderings of CT scans of the mandibles of the three individuals used in this study showing locations, relative size, and orientations of the delta rosette strain gages. The lateral gages were placed $5 \mathrm{~mm}$ rostral to the insertion of the superficial masseter, one at midcorpus height and one near the bottom edge of the corpus. This location corresponds to the lateral mandibular prominence in hominids where the root of the ramus merges with the corpus (Brown, 1997; Kimbel et al., 2004). The medial gage was placed inferior to the attachment of the mylohyoid muscle. Note that gages were placed on the right corpus during experiments 183 (animal M) and 184 (animal C), and on the left corpus in Experiment 185 (animal S). Gage positions and orientations relative to the occlusal plane were determined from radiographs taken following recording.

Figure 3. Average mandible kinematics through a gape cycle comparing control (no gages, dashed lines) and experimental (with gages, solid lines) conditions for two of the experimental subjects. Plots indicate the angular velocity of the mandible with respect to the fixed cranium. Blue traces show the rotation around the X-axis (see inset), indicating jaw opening and closing (positive values for jaw opening and negative values for jaw closing), while green traces show the rotation around the Z-axis, indicating lateral deviation (positive for a mandible moving towards the working side and negative for a mandible moving towards the balancing side). Top panels show the mandible kinematics of experimental subject $\mathrm{C}$ for a time-standardized gape cycle (A) and in absolute time, centered around maximum gape (B). Bottom panels show the mandible kinematics of experimental subject $S$ for a time-standardized gape cycle $(C)$ and in absolute time, centered around maximum gape (D). Kinematic traces are presented as mean values and their respective standard errors of the mean (lines and shadow traces around them, respectively). Gray sections indicate the jaw-closing phase of the gape cycle.

Figure 4. Mean $\varepsilon_{1}$ orientation and magnitude recorded from the upper lateral gage during feeding by animal $M$ in Experiment 183. Red lines are vectors illustrating orientation and magnitude of $\varepsilon_{1}$. A) Mean $\varepsilon_{1}$ orientations recorded during different behaviors. Gray lines in $A$ illustrate mean orientations where low magnitudes make it difficult to distinguish the vectors. B) Mean $\varepsilon_{1}$ orientations during left (balancing side) chews, with each vector representing a 
different food type. C) Mean $\varepsilon_{1}$ orientations during right (working side) chews, with each vector representing a different food type. Black arrows illustrate mean $\varepsilon_{1}$ orientations during all left chews (B) and all right chews (C).

Figure 5. Mean $\varepsilon_{1}$ orientation and magnitude recorded from the lower lateral gage during feeding by animal $M$ in Experiment 183. Red lines are vectors illustrating orientation and magnitude of $\varepsilon_{1}$. A) Mean $\varepsilon_{1}$ orientations recorded during different behaviors. Gray lines in $A$ illustrate mean orientations where low magnitudes make it difficult to distinguish the vectors. B) Mean $\varepsilon_{1}$ orientations during left (balancing side) chews, with each vector representing a different food type. C) Mean $\varepsilon_{1}$ orientations during right (working side) chews, with each vector representing a different food type. Black arrows illustrate mean $\varepsilon_{1}$ orientations during all left chews (B) and all right chews (C).

Figure 6. Mean $\varepsilon_{1}$ orientation and magnitude recorded from the medial gage during feeding by animal $M$ in Experiment 183. Red lines are vectors illustrating orientation and magnitude of $\varepsilon_{1}$. A) Mean $\varepsilon_{1}$ orientations recorded during different behaviors. Gray lines in A illustrate mean orientations where low magnitudes make it difficult to distinguish the vectors. B) Mean $\varepsilon_{1}$ orientations during left (balancing side) chews, with each vector representing a different food type. C) Mean $\varepsilon_{1}$ orientations during right (working side) chews, with each vector representing a different food type. Black arrows illustrate mean $\varepsilon_{1}$ orientations during all left chews (B) and all right chews (C).

Figure 7. Mean $\varepsilon_{1}$ orientation and magnitude recorded from the upper lateral gage during feeding by animal $C$ in Experiment 184. Red lines are vectors illustrating orientation and magnitude of $\varepsilon_{1}$. A) Mean $\varepsilon_{1}$ orientations recorded during different behaviors. Gray lines in $A$ illustrate mean orientations where low magnitudes make it difficult to distinguish the vectors. B) Mean $\varepsilon_{1}$ orientations during left (balancing side) chews, with each vector representing a different food type. C) Mean $\varepsilon_{1}$ orientations during right (working side) chews, with each vector representing a different food type. Black arrows illustrate mean $\varepsilon_{1}$ orientations during all left chews (B) and all right chews (C).

Figure 8. Mean $\varepsilon_{1}$ orientation and magnitude recorded from the lower lateral gage during feeding by animal $\mathrm{C}$ in Experiment 184. Red lines are vectors illustrating orientation and magnitude of $\varepsilon_{1}$. A) Mean $\varepsilon_{1}$ orientations recorded during different behaviors. Gray lines in $A$ illustrate mean orientations where low magnitudes make it difficult to distinguish the vectors. B) Mean $\varepsilon_{1}$ orientations during left (balancing side) chews, with each vector representing a different food type. C) Mean $\varepsilon_{1}$ orientations during right (working side) chews, with each vector 
representing a different food type. Black arrows illustrate mean $\varepsilon_{1}$ orientations during all left chews (B) and all right chews (C).

Figure 9. Mean $\varepsilon_{1}$ orientation and magnitude recorded from the medial gage during feeding by animal $C$ in Experiment 184. Red lines are vectors illustrating orientation and magnitude of $\varepsilon_{1}$. A) Mean $\varepsilon_{1}$ orientations recorded during different behaviors. Gray lines in A illustrate mean orientations where low magnitudes make it difficult to distinguish the vectors. B) Mean $\varepsilon_{1}$ orientations during left (balancing side) chews, with each vector representing a different food type. C) Mean $\varepsilon_{1}$ orientations during right (working side) chews, with each vector representing a different food type. Black arrows illustrate mean $\varepsilon_{1}$ orientations during all left chews (B) and all right chews $(\mathrm{C})$.

Figure 10. Mean $\varepsilon_{1}$ orientation and magnitude recorded during feeding by animal $\mathrm{S}$ in Experiment 185. Red lines are vectors illustrating orientation and magnitude of $\varepsilon_{1}$. Gray lines illustrate mean orientations where low magnitudes make it difficult to distinguish the vectors. A) Mean $\varepsilon_{1}$ orientations recorded from the upper lateral gage during different behaviors. B) Mean $\varepsilon_{1}$ orientations recorded from the lower lateral gage during different behaviors. C) Mean $\varepsilon_{1}$ orientations recorded from the medial gage during different behaviors.

Figure 11. Mean (box) and maximum (whisker) $\varepsilon_{1}$ strain magnitudes recorded during the three experiments, separated by behavior. Strains varied by behavior, with the largest strains at all gage sites being during biting and not during chewing.

Figure 12. A) Food material and geometric properties can only impact mandible morphology by eliciting variation in feeding behaviors that in turn result in differences in mandibular loading and strain regimes. B) The relative importance of these different behaviors for mandible morphology depends on weighting factors, including biomechanical variables such as strain magnitude and the amount of time or number of times the mandible is subjected to a given strain regime. A range of ecological variables can also weight the importance of the behavior, such as whether the resource is necessary for survival (e.g., a fall-back food). 


\section{References}

Beecher, R.M., 1977. Function and fusion at the mandibular symphysis. Am. J. Phys. Anthropol. 47, 325-336.

Beecher, R.M., 1979. Functional significance of the mandibular symphysis. J. Morphol. 159, 117-130.

Bouvier, M., 1986a. A biomechanical analysis of mandibular scaling in Old World monkeys. Am. J. Phys. Anthropol. 69, 473-482.

Bouvier, M., 1986b. Biomechanical scaling of mandibular dimensions in New World monkeys. Int. J. Primatol. 7, 551-567.

Bouvier, M., Hylander, W.L., 1981. Effect of bone strain on cortical bone structure in macaques (Macaca mulatta). J. Morphol. 167, 1-12.

Bouvier, M., Hylander, W.L., 1994. Bone remodeling responses to strain gradients in the zygomatic arch of macaques. J. Dent. Res. 73, 195-195.

Bouvier, M., Hylander, W.L., 1996a. The mechanical or metabolic function of secondary osteonal bone in the monkey Macaca fascicularis. Archs. Oral Biol. 41(10), 941-950.

Bouvier, M., Hylander, W.L., 1996b. Strain gradients, age, and levels of modeling and remodeling in the facial bones of Macaca fascicularis. In: Davidovitch, Z., Norton, L.A. (Eds.), Biological Mechanisms of Tooth Movement and Craniofacial Adaptation. Harvard Society for the Advancement of Orthodontics, Boston, pp. 407-412.

Brown, B., 1997. Miocene hominoid mandibles-functional and phylogenetic perspectives., in: Begun, D.R., Ward, C.V., Rose, M.D. (Eds.), Function, Phylogeny and Fossils: Miocene Hominoid Evolution and Adaptation. Plenum, New York, pp. 153-171.

Burstein, A.H., Currey, J.D., Frankel, V.H., Reilly, D.T., 1972. The ultimate properties of bone tissue: the effects of yielding. J. Biomech 5, 35-44.

Carter, D.R., Hayes, W.C., Schurman, D.J., 1976. Fatigue life of compact bone-II. Effects of microstructure and density. J. Biomech. 9, 211-214. 
Carter, D.R., Harris, W.H., Vasu, R., Caler, W.E., 1981. The mechanical and biological response of cortical bone to in vivo strain histories. In: Cowin, S.C. (Ed.), Mechanical Properties of Bone (AMD vol. 45). American Society of Mechanical Engineers, New York, pp. 81-92.

Currey, J.D., 2004. Tensile yield in compact bone is determined by strain, post-yield behaviour by mineral content. J. Biomech. 37, 549-556.

Daegling, D.J., 1989. Biomechanics of cross-sectional size and shape in the hominoid mandibular corpus. Am. J. Phys. Anthropol. 80, 91-106.

Daegling, D.J., 1990. Geometry and biomechanics of hominoid mandibles. Ph.D. Dissertation, State University of New York at Stony Brook.

Daegling, D.J., 1992. Mandibular morphology and diet in the genus Cebus. Int. J. Primatol. 13, 545-570.

Daegling, D.J., 1993. The relationship of in vivo bone strain to mandibular corpus morphology in Macaca fascicularis. J. Hum. Evol. 25, 247-269.

Daegling, D.J., 2002. Bone geometry in cercopithecoid mandibles. Archs. Oral Biol. 47, 315-325.

Daegling, D.J., 2007. Relationship of bone utilization and biomechanical competence in hominoid mandibles. Archs. Oral Biol. 52, 51-63.

Daegling, D.J., 2012. The human mandible and the origins of speech. J. Anthropol. 2012, doi:10.1155/2012/2015024.

Daegling, D.J., Grine, F.E., 2006. Mandibular biomechanics and the paleontological evidence for the evolution of human diet. In: Ungar, P.S. (Ed.), Evolution of the Human Diet : The Known, the Unknown, and the Unknowable. Oxford University Press, Cary, pp. 77-105.

Daegling, D.J., McGraw, W.S., 2001. Feeding, diet, and jaw form in West African Colobus and Procolobus. Int. J. Primatol. 22, 1033-1055.

Daegling, D.J., McGraw, W.S., 2007. Functional morphology of the mangabey mandibular corpus: Relationship to dental specializations and feeding behavior. Am. J. Phys. Anthropol. 134, 50-62. 
Demes, B., 1998. Use of strain gauges in the study of primate locomotor biomechanics. In: Strasser, E., Fleagle, J., Rosenberger, A., McHenry, H. (Eds.), Primate Locomotion. Recent Advances. Plenum Press, New York, pp. 237-254.

Demes, B., 2007. In vivo bone strain and bone functional adaptation. Am. J. Phys. Anthropol. $133,717-722$.

Demes, B., Qin, Y.X., Stern, J.T., Larson, S.G., Rubin, C.T., 2001. Patterns of strain in the macaque tibia during functional activity. Am. J. Phys. Anthropol. 116, 257-265.

Ehrich, T.H., Vaughn, T.T., Koreishi, S.F., Linsey, R.B., Pletscher, L.S., Cheverud, J.M., 2003. Pleiotropic effects on mandibular morphology I. Developmental morphological integration and differential dominance. J. Exp. Zool. B Mol. Dev. Evol. 296, 58-79.

Ehrich, T.H., Kenney-Hunt, J.P., Pletscher, L.S., Cheverud, J.M., 2005. Genetic variation and correlation of dietary response in an advanced intercross mouse line produced from two divergent growth lines. Genet. Res. 85, 211-222.

Fisher, N.I., 1993. Statistical analysis of circular data. Cambridge University Press, New York.

Forwood, M.R., Turner, C.H., 1995. Skeletal adaptations to mechanical usage: Results from tibial loading studies in rats. Bone 17, 197S-205S.

Frost, H.M., 1987. The mechanostat: a proposed pathogenic mechanism of osteoporoses and the bone mass effects of mechanical and nonmechanical agents. Bone Min. 2, 73-85.

Frost, H.M., 2000. Why the ISMNI and the Utah paradigm? Their role in skeletal and extraskeletal disorders. J. Musculoskel. Neuronal Interact. 1, 5-9.

Hiiemae, K.M., 2000. Feeding in mammals. In: Schwenk, K. (Ed.), Feeding: Form, Function and Evolution in Tetrapod Vertebrates. Academic Press, London, pp. 411-457.

Hylander, W.L., 1979a. An experimental analysis of temporomandibular joint reaction force in macaques. Am. J. Phys. Anthropol. 51, 433-456.

Hylander, W.L., 1979b. The functional significance of primate mandibular form. J. Morphol. 160, 223-240. 
Hylander, W.L., 1979c. Mandibular function in Galago crassicaudatus and Macaca fascicularis: An in vivo approach to stress analysis of the mandible. J. Morphol. 159, 253-296.

Hylander, W.L., 1984. Stress and strain in the mandibular symphysis of primates: A test of competing hypotheses. Am. J. Phys. Anthropol. 64, 1-46.

Hylander, W.L., 1988. Implications of in vivo experiments for interpreting the functional significance of "robust" australopithecine jaws. In: Grine, F.E. (Ed.), Evolutionary History of the "Robust" Australopithecines. Aldine de Gruyter, New York, pp. 55-83.

Hylander, W.L., 2013. Functional links between canine height and jaw gape in catarrhines with special reference to early hominins. Am. J. Phys. Anthropol. 150, 247-259.

Hylander, W.L., Bays, R., 1979. In vivo strain gauge analysis of the dentary squamosal joint reaction force during mastication and incisal biting in Macaca mulatta and Macaca fascicularis. Archs. Oral Biol. 24, 689-697.

Hylander, W.L., Johnson, K.R., 1994. Jaw muscle function and wishboning of the mandible during mastication in macaques and baboons. Am. J. Phys. Anthropol. 94, 523-547.

Hylander, W.L., Johnson, K.R., Crompton, A.W., 1987. Loading patterns and jaw movements during mastication in Macaca fascicularis: A bone-strain, electromyographic, and cineradiographic analysis. Am. J. Phys. Anthropol. 72, 287-314.

Iriarte-Diaz, J., Reed, D.A., Ross, C.F., 2011. Sources of variance in temporal and spatial aspects of jaw kinematics in two species of primates feeding on foods of different properties. Integr. Comp. Biol. 51, 307-319.

Kay, R.F., Cartmill, M., 1977. Cranial morphology and adaptations of Palaechthon nacimienti and other Paromomyidae (Plesiadapoidea? Primates), with a description of a new genus and species. J. Hum. Evol. 6, 19-35.

Kimbel, W.H., Johanson, D., Rak, Y., 2004. The Skull of Australopithecus afarensis. Oxford University Press, Oxford.

Lynch-Alfaro, J.W., Silva, J.D.E., Rylands, A.B., 2012. How different are robust and gracile capuchin monkeys? An argument for the use of Sapajus and Cebus. Am. J. Primatol. 74, 273286. 
Mardia, K.V., Jupp, P.E., 2000. Directional Statistics. Wiley, Chichester.

Ravosa, M.J., 1991. Structural allometry of the mandibular corpus and symphysis in prosimian primates. J. Hum. Evol. 20, 3-20.

Ravosa, M.J., 1992. Allometry and heterochrony in extant and extinct Malagasy primates. J. Hum. Evol. 23, 197-217.

Ravosa, M.J., 1996. Jaw morphology and function in living and fossil Old World Monkeys. Int. J. Primatol. 17, 909-932.

Ravosa, M.J., 2000. Size and scaling in the mandible of living and extinct apes. Folia Primatol. 71, 305-322.

Ravosa, M.J., 2007. Cranial ontogeny, diet, and ecogeographic variation in African lorises. Am. J. Primatol. 69, 59-73.

Ravosa, M.J., Nicholson, E.K., Stock, S.R., Hamrick, M.W., 2006. Biomineralization and adaptive plasticity of the temporomandibular joint in myostatin knockout mice. Archs. Oral Biol. 51, 3749.

Ravosa, M.J., Klopp, E.B., Pinchoff, J., Stock, S.R., Hamrick, M.W., 2007a. Plasticity of mandibular biomineralization in myostatin-deficient mice. J. Morphol. 268, 275-282.

Ravosa, M.J., Kunwar, R., Stock, S.R., Stack, M.S., 2007b. Pushing the limit: masticatory stress and adaptive plasticity in mammalian craniomandibular joints. J. Exp. Biol. 210, 628-641.

Reed, D.A., Ross, C.F., 2010. The influence of food material properties on jaw kinematics in the primate, Cebus. Archs. Oral Biol. 55, 946-962.

Reilly, D.T., Burstein, A.H., 1974. Review article. The mechanical properties of cortical bone. J. Bone Joint Surg. 56, 1001-1022.

Reilly, D.T., Burstein, A.H., 1975. The elastic and ultimate properties of compact bone tissue. J. Biomech. 8, 393-405. 
Reilly, G.C., Currey, J.D., 1999. The development of microcracking and failure in bone depends on the loading mode to which it is adapted. J. Exp. Biol. 202, 543-552.

Ross, C.F., Iriarte-Diaz, J., 2014. What does feeding system morphology tell us about feeding? Evol. Anthropol. 23, 105-120.

Ross, C.F., Metzger, K.A., 2004. Bone strain gradients and optimization in tetrapod skulls. Ann. Anat. 186, 387-396.

Ross, C.F., Lockwood, C.A., Fleagle, J.G., Jungers, W.L., 2002. Adaptation and behavior in the primate fossil record. In: Plavcan, J.M., Kay, R.F., Jungers, W.L., van Schaik, C.P. (Eds.), Reconstructing Behavior in the Primate Fossil Record. Kluwer Academic/Plenum Publishers, New York, pp. 1-41.

Ross, C.F., Iriarte-Diaz, J., Nunn, C.L., 2012. Innovative approaches to the relationship between diet and mandibular morphology in primates. Int. J. Primatol. 33, 632-660.

Rybicki, E.F., Mills, E.J., Turner, A.S., Simonen, F.A., 1977. In vivo and analytical studies of forces and moments in equine long bones. J. Biomech. 10, 701-705.

Smith, R.J., 1984. Comparative functional morphology of maximum mandibular opening (gape) in primates. In: Chivers, D.J., Wood, B.A., Bilsborough, A. (Eds.), Food Acquisition and Processing in Primates. Plenum Press, New York, pp. 231-255.

Taylor, A.B., 2002. Masticatory form and function in the African apes. Am. J. Phys. Anthropol. $117,133-156$.

Taylor, A.B., 2006a. Diet and mandibular morphology in African apes. Int. J. Primatol. 27, 181201.

Taylor, A.B., 2006b. Feeding behavior, diet, and the functional consequences of jaw form in orangutans, with implications for the evolution of Pongo. J. Hum. Evol. 50, 377-393.

Taylor, A.B., Vogel, E.R., Dominy, N.J., 2008. Food material properties and mandibular load resistance abilities in large-bodied hominoids. J. Hum. Evol. 55, 604-616. 
Terhune, C.E., Hylander, W.L., Vinyard, C.J., Taylor, A.B., 2011. Masseter fiber length and position influence relative maximum jaw gapes in the sexually-dimorphic Macaca fascicularis. Am. J. Phys. Anthropol. 144, 292-292.

Theriault, B.R., Reed, D.A., Niekrasz, M.A., 2008. Reversible medetomidine/ketamine anesthesia in captive capuchin monkeys (Cebus apella). J. Med. Primatol. 37, 74-81.

Turner, C.H., 1998. Three rules for bone adaptation to mechanical stimuli. Bone 23, 399-407.

Van Soest, P.J., 1996. Allometry and ecology of feeding behavior and digestive capacity in herbivores: A review. Zoo Biol. 15, 455-479.

Vinyard, C.J., Ryan, T.M., 2006. Cross-sectional bone distribution in the mandibles of gouging and non-gouging Platyrrhini. Int. J. Primatol. 27, 1461-1490.

Vinyard, C.J., Schmitt, D., 2004. New technique for studying reaction forces during primate behaviors on vertical substrates. Am. J. Phys. Anthropol. 125, 343-351.

Vinyard, C.J., Wall, C.E., Williams, S.H., Schmitt, D., Hylander, W.L., 2001. A preliminary report on the jaw mechanics during tree gouging in common marmosets (Callithrix jacchus). In: Brooks, A. (Ed.), Dental morphology 2001: Proceedings of the 12th International Symposium on Dental Morphology. Sheffield Academic Press, Sheffield, pp. 283-297.

Vinyard, C.J., Wall, C.E., Williams, S.H., Hylander, W.L., 2003. Comparative functional analysis of skull morphology of tree-gouging primates. Am. J. Phys. Anthropol. 120, 153-170.

Vinyard, C.J., Wall, C.E., Williams, S.H., Hylander, W.L., 2008. Patterns of variation across primates in jaw-muscle electromyography during mastication. Integr. Comp. Biol. 48, 294-311.

Vinyard, C.J., Wall, C.E., Williams, S.H., Mork, A.L., Armfield, B.A., Melo, L.C.D., ValencaMontenegro, M.M., Valle, Y.B.M., de Oliveira, M.A.B., Lucas, P.W., Schmitt, D., Taylor, A.B., Hylander, W.L., 2009. The Evolutionary Morphology of Tree Gouging in Marmosets. In: Ford, S.M., Porter, L.M., Davis, L.C. (Eds.), The Smallest Anthropoids: The Marmoset/Callimico Radiation. Springer, New York, 395-409.

Vogel, E.R., Zulfa, A., Hardus, M., Wich, S.A., Dominy, N.J., Taylor, A.B., 2014. Food mechanical properties, feeding ecology, and the mandibular morphology of wild orangutans. J. Hum. Evol. $75,110-124$. 
Wallisch, P., Lusignan, M., Benayoun, M., Baker, T.I., Dickey, A.S., Hatsopoulos, N.G., 2009. Matlab (R) for Neuroscientists: An Introduction to Scientific Computing in Matlab (R). Elsevier, San Diego.

Weijs, W.A., De Jong, J.H., 1977. Strain in mandibular alveolar bone during mastication in the rabbit. Archs. Oral Biol. 22, 667-675.

Williams, S.H., Stover, K.K., Davis, J.S., Montuelle, S.J., 2011. Mandibular corpus bone strains during mastication in goats (Capra hircus): A comparison of ingestive and rumination chewing. Archs. Oral Biol. 56, 960-971.

Woltring, H.J., Huiskes, R., de Lange, A., Veldpaus, F.E., 1985. Finite centroid and helical axis estimation from noisy landmark measurements in the study of human joint kinematics. J. Biomech. 18, 379.

Wright, B.W., 2004. Food mechanical properties and niche partitioning in a community of Neotropical primates. Am. J. Phys. Anthropol. 212-212.

Wright, B.W., 2005a. Craniodental biomechanics and dietary toughness in the genus Cebus. J. Hum. Evol. 48, 473-492.

Wright, B.W., 2005b. Dietary demand and niche breadth among six primates in Guyana, South America. Am. J. Phys. Anthropol., Suppl. 40, 227.

Wright, K.A., Wright, B.W., Ford, S.M., Fragaszy, D., Izar, P., Norconk, M., Masterson, T., Hobbs, D.G., Alfaro, M.E., Alfaro, J.W.L., 2015. The effects of ecology and evolutionary history on robust capuchin morphological diversity. Mol. Phylogenet. Evol. 82, 455-466.

Zioupos, P., Xiao, T.W., Currey, J.D., 1996. Experimental and theoretical quantification of the development of damage in fatigue tests of bone and antler. J. Biomech. 29, 989-1002.

Zioupos, P., Currey, J.D., Casinos, A., 2001. Tensile fatigue in bone: Are cycles-, or time to failure, or both, important? J. Theor. Biol. 210, 389. 
Table 1. Terminology and abbreviations.

\section{Term}

Loading regime

Deformation regime

\section{Stress regime}

Strain regime

FMP

E

$R$

$\varepsilon_{1}$

$\varepsilon_{2}$

$\gamma_{\max }$

\section{Definition}

Combination of external forces acting on the mandible

Pattern of deformation associated with a loading regime

Pattern of internal forces at local points associated with a loading

$$
\text { regime }
$$

Pattern of strains (principal, shear, Von Mises) at local points associated with a loading regime

Food material properties

$$
\begin{aligned}
& \text { Young's modulus } \\
& \text { Toughness }
\end{aligned}
$$

Maximum principal strain

Minimum principal strain

Shear strain

\section{Examples}

Muscle, joint, and bite forces

Bending, twisting

Principal, shear, Von Mises stress

Principal, shear, Von Mises strain

$$
\begin{aligned}
& \text { Units } \\
& \mathrm{MPa} \\
& \mathrm{J} \mathrm{m}^{-2}
\end{aligned}
$$

Microstrain $(\mu \varepsilon)$

Microstrain $(\mu \varepsilon)$

Microstrain $(\mu \varepsilon)$ 


\section{figure 1}

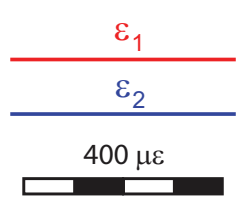

TW, transducer bite ipsilateral to gage TB, transducer bite contralateral to gage

$\mathrm{TI}$, transducer bite incisors $\mathrm{CW}$, apple chew ipsilateral to gage $\mathrm{CB}$, apple chew contralateral to gage I, apple incisor bite

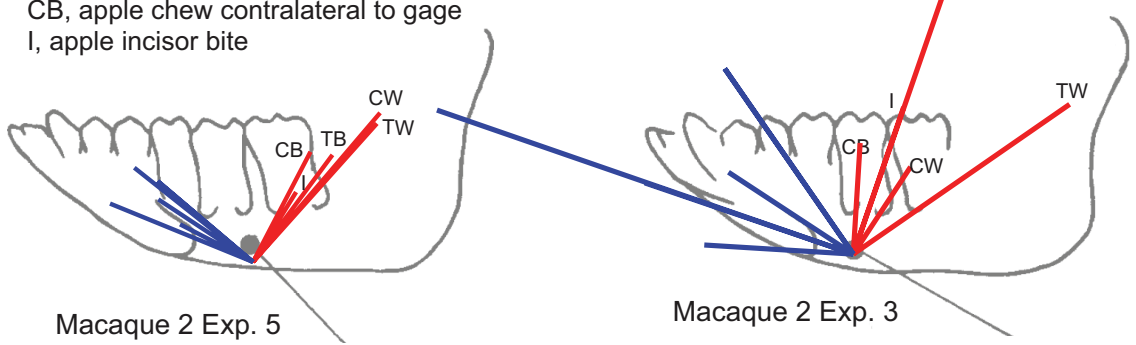

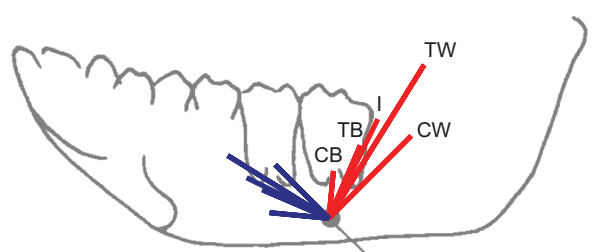

Macaque 3 Exp. 6

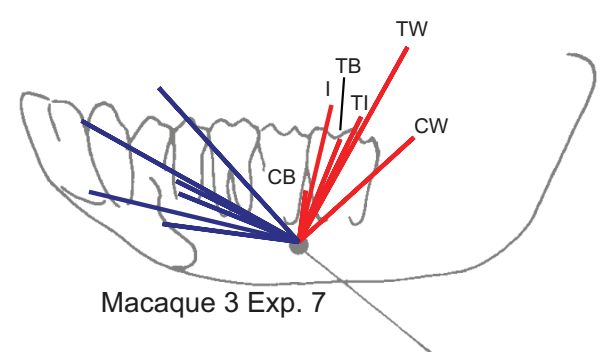

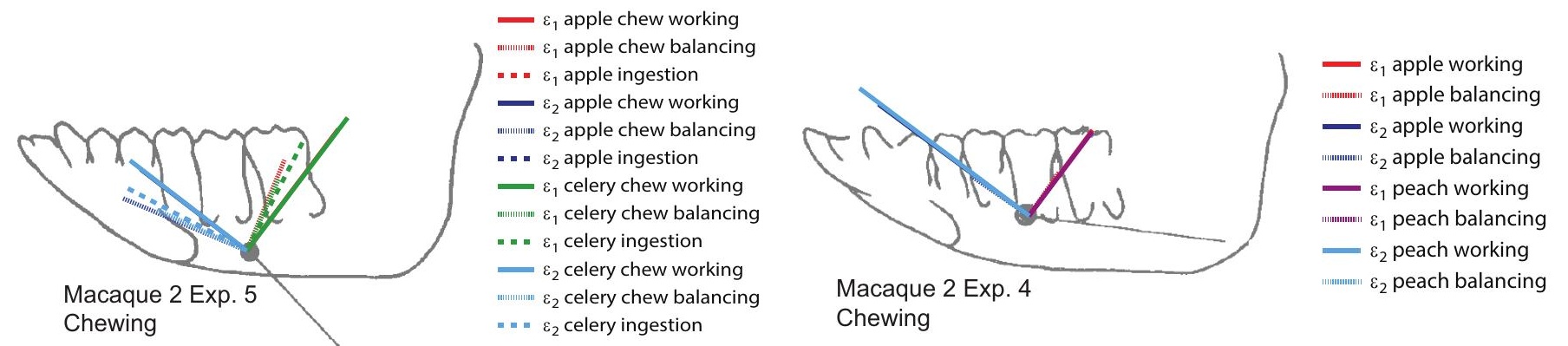


Experiment 183 - Animal M

Lateral

Lingual

figure 2

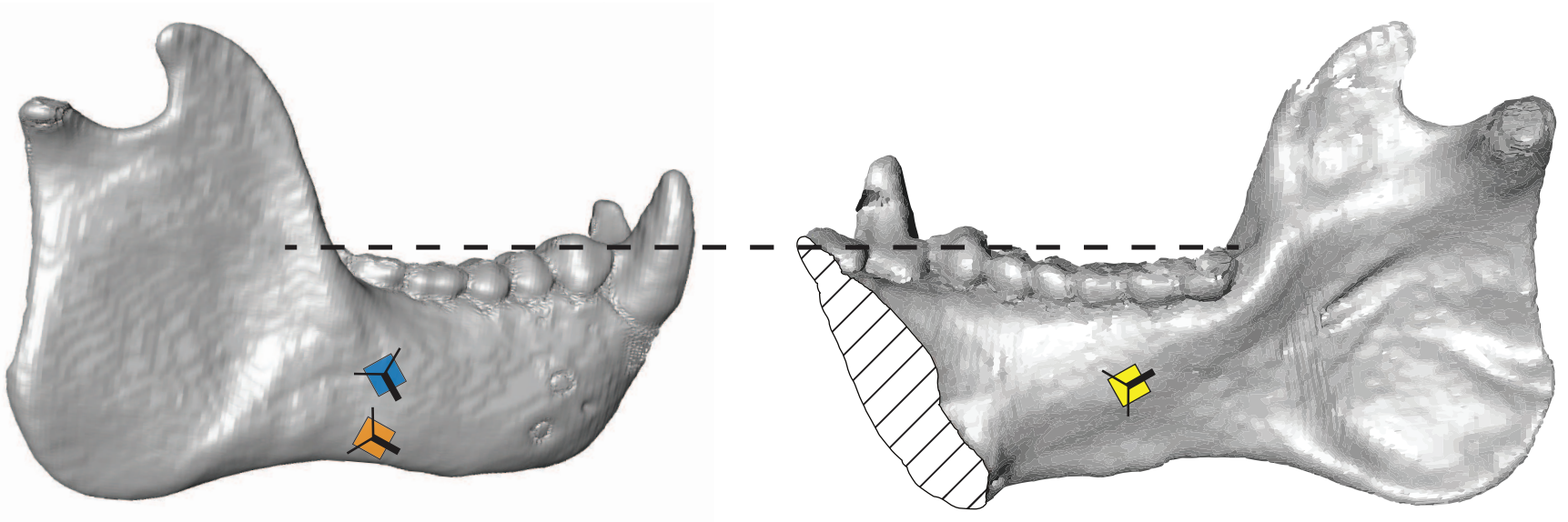

Experiment 184 - Animal C

Lateral

Lingual

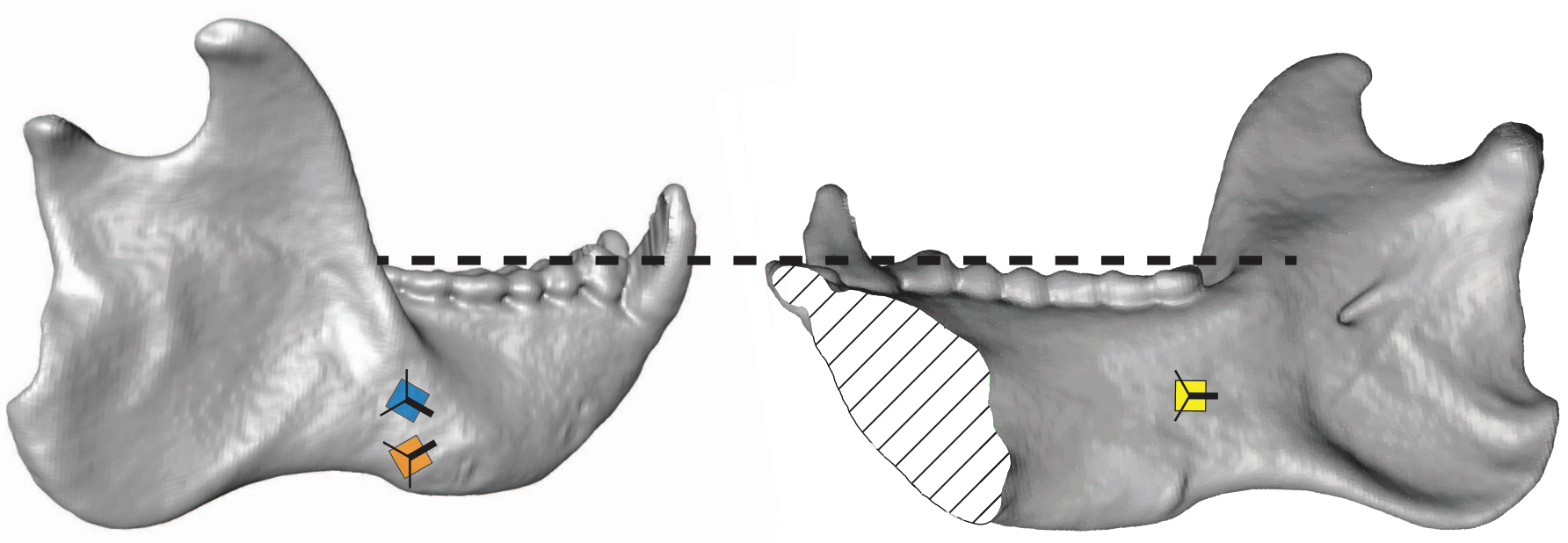

Experiment 185 - Animal S

Lateral

Lingual

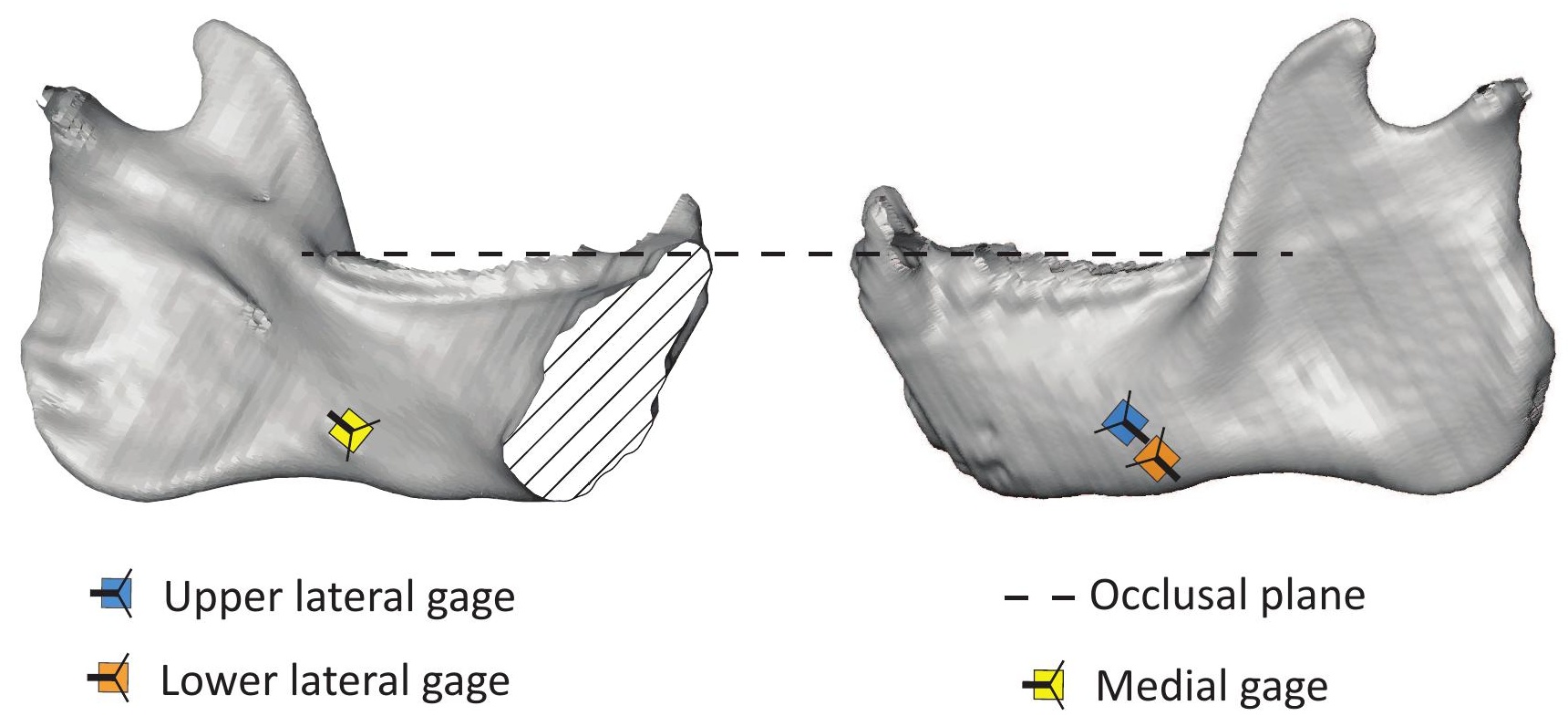



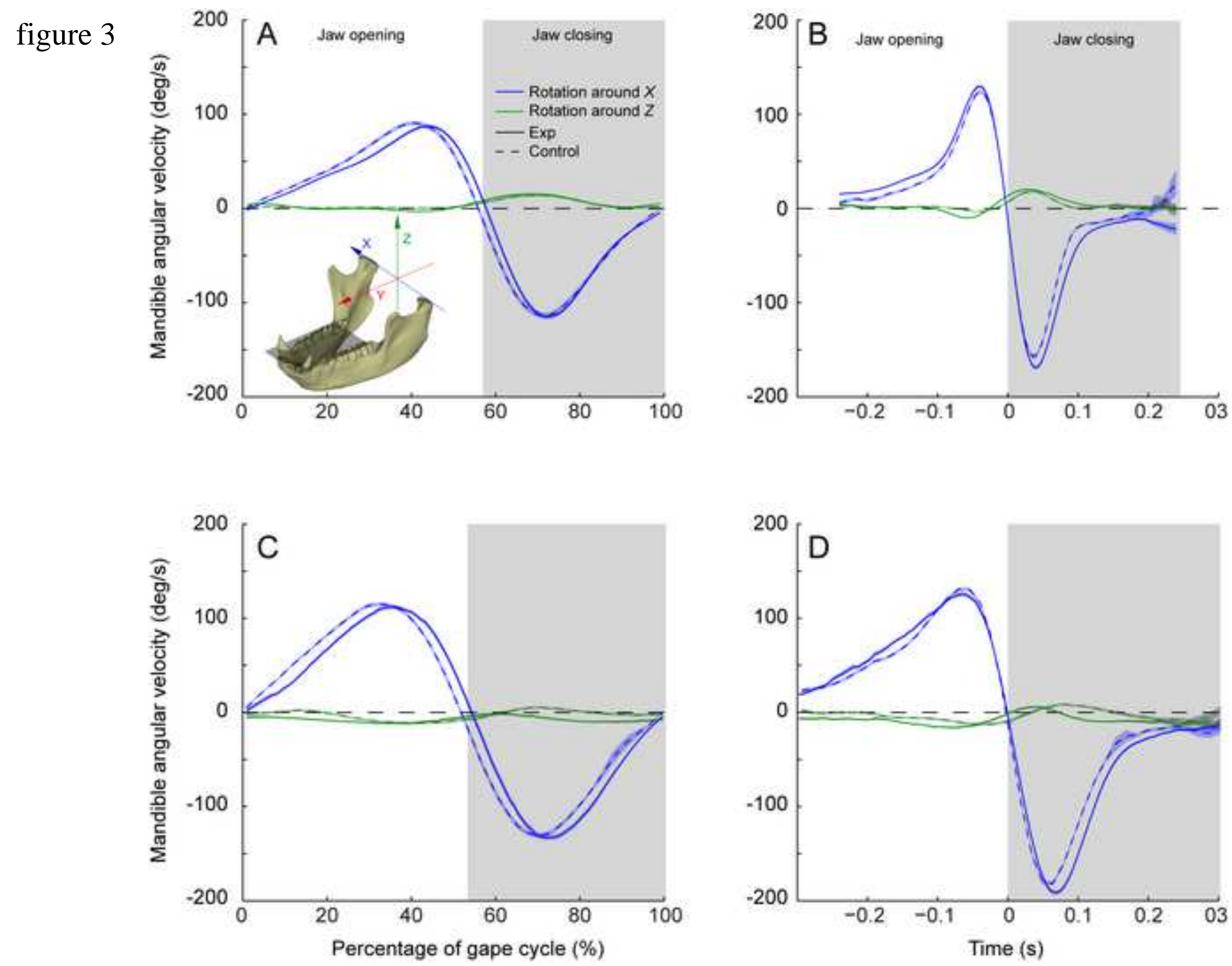
figure 4
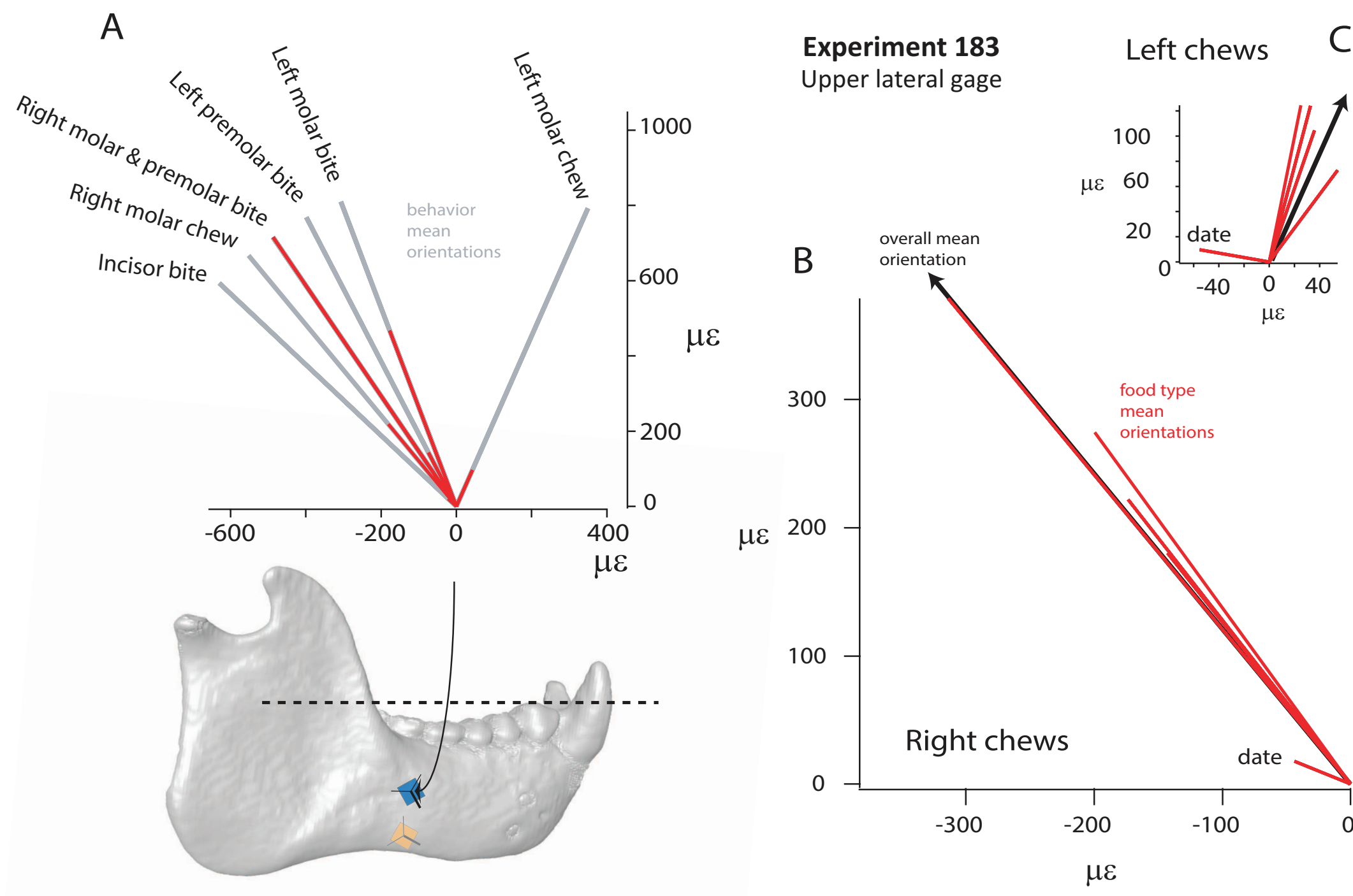
figure 5

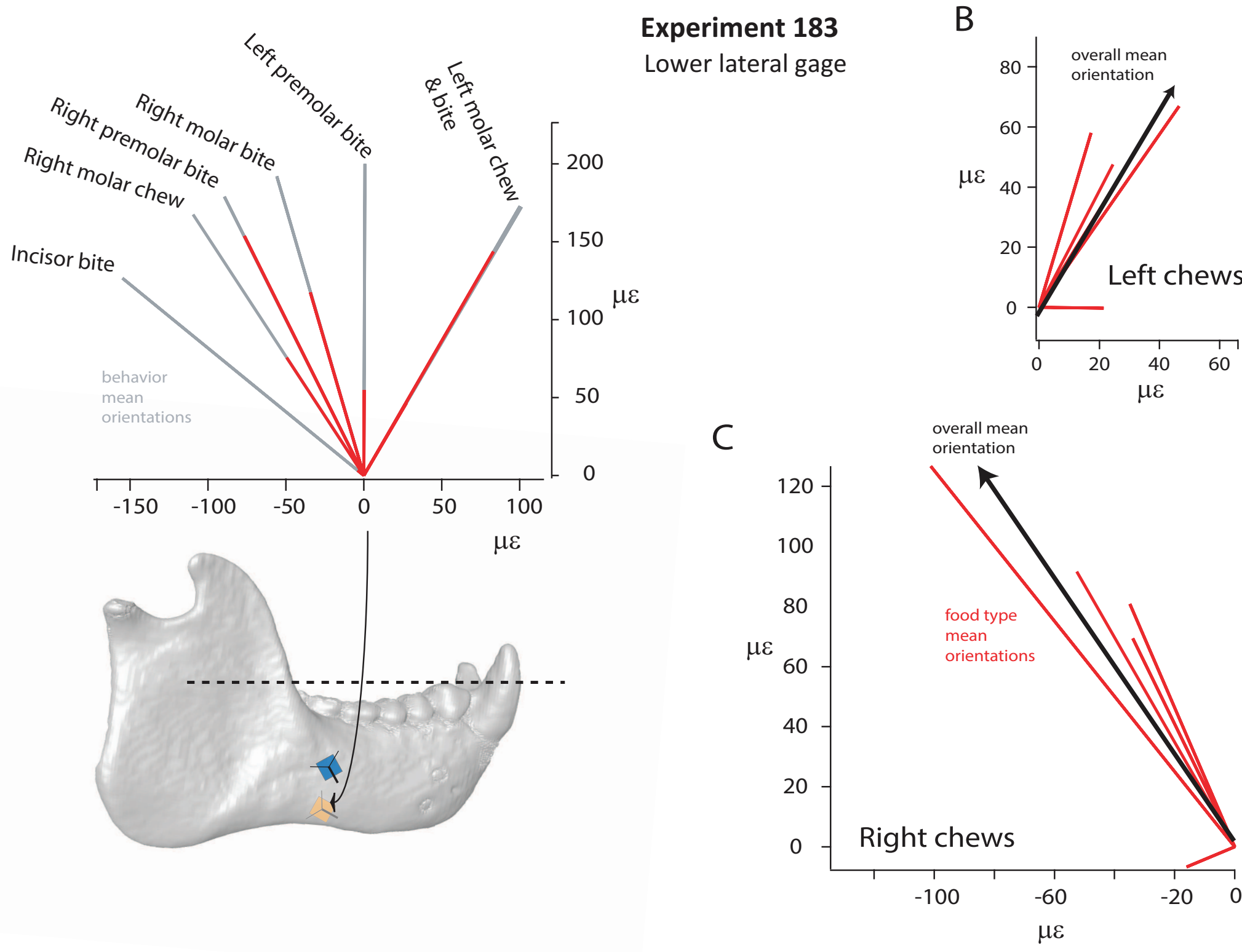




\section{figure 6}

A
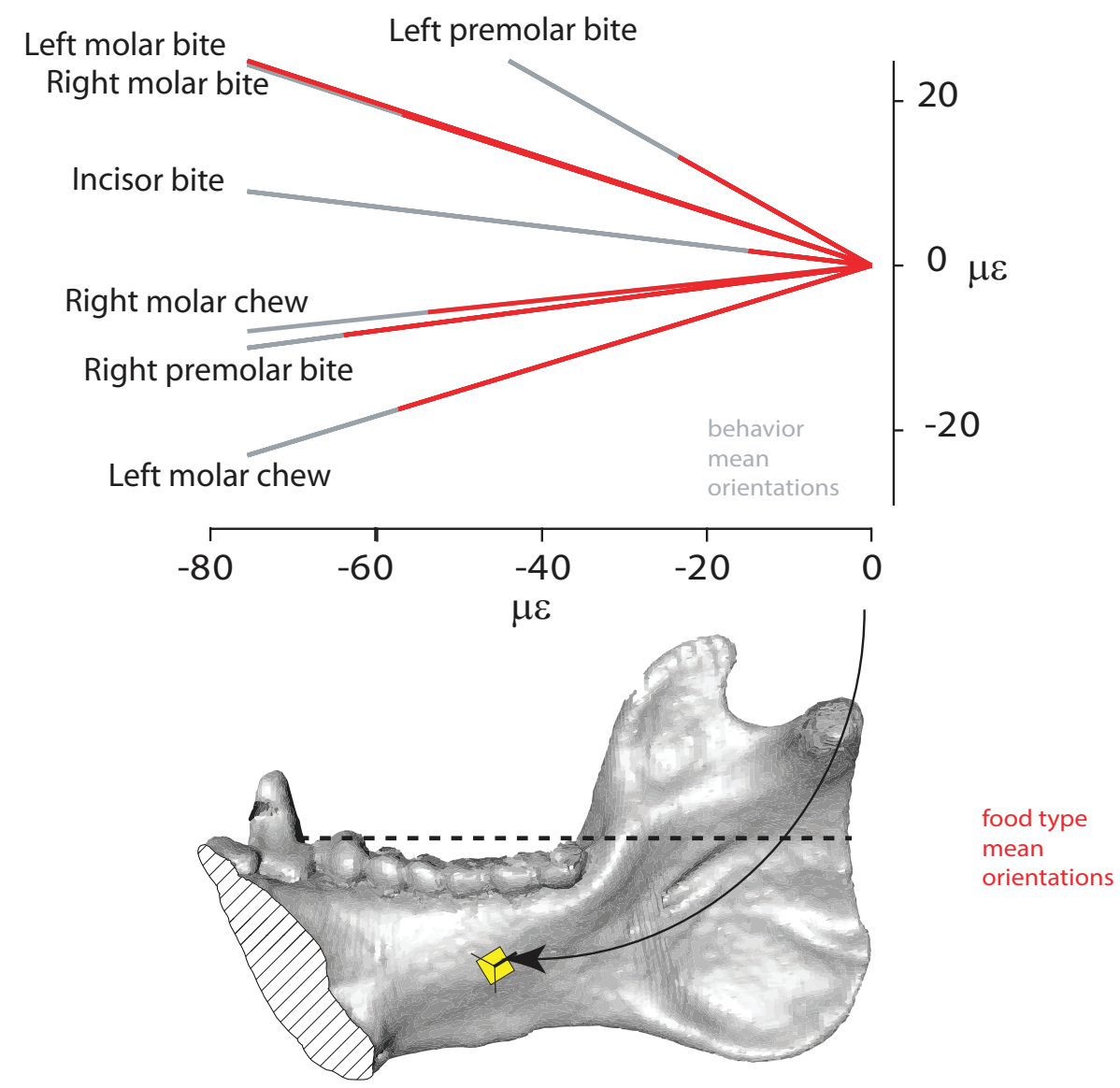

B
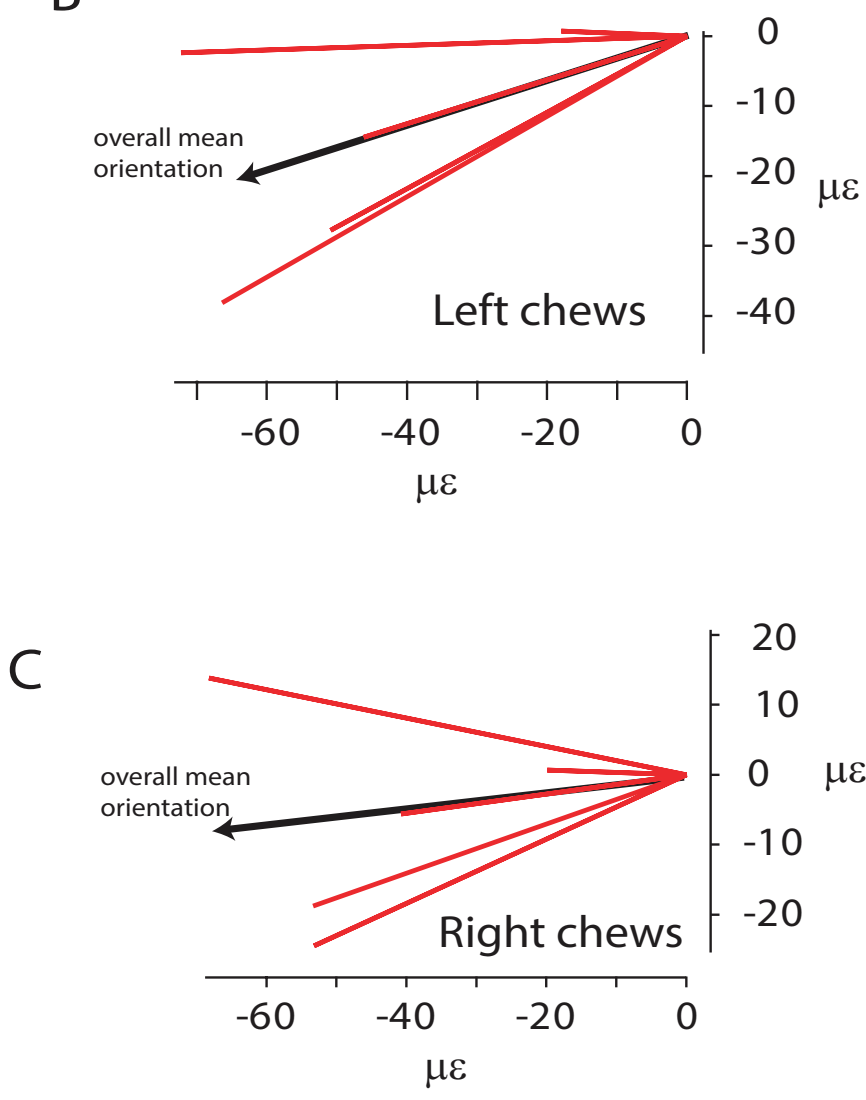


\section{figure 8}

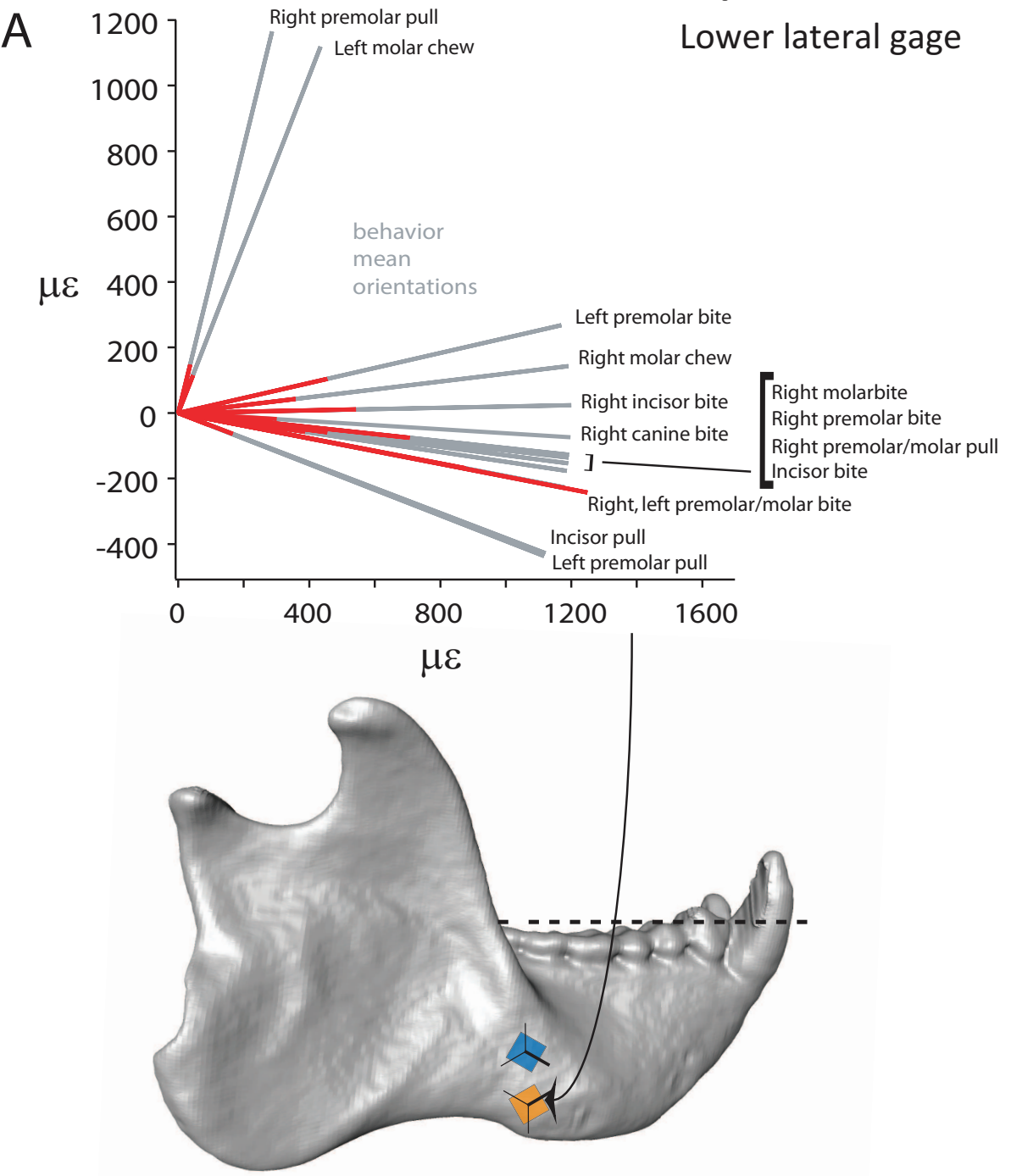

\section{Experiment 184}

\section{Lower lateral gage}

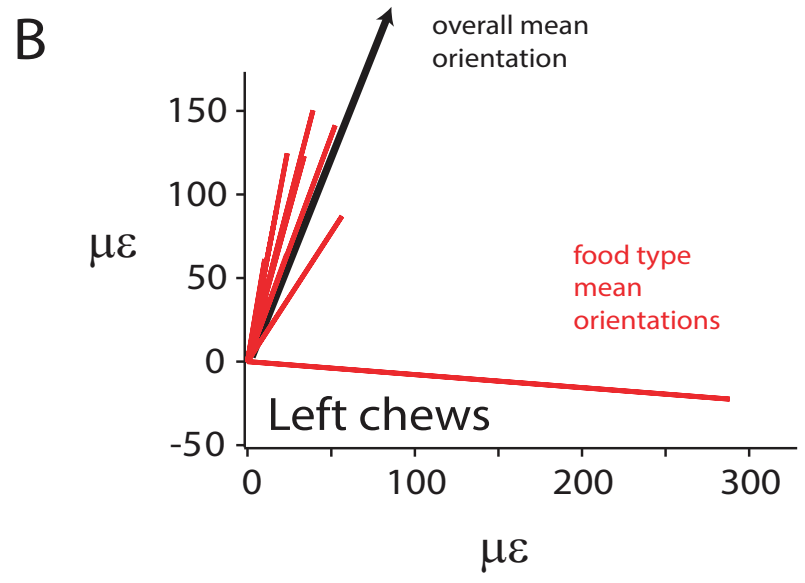

C

$\mu \varepsilon$

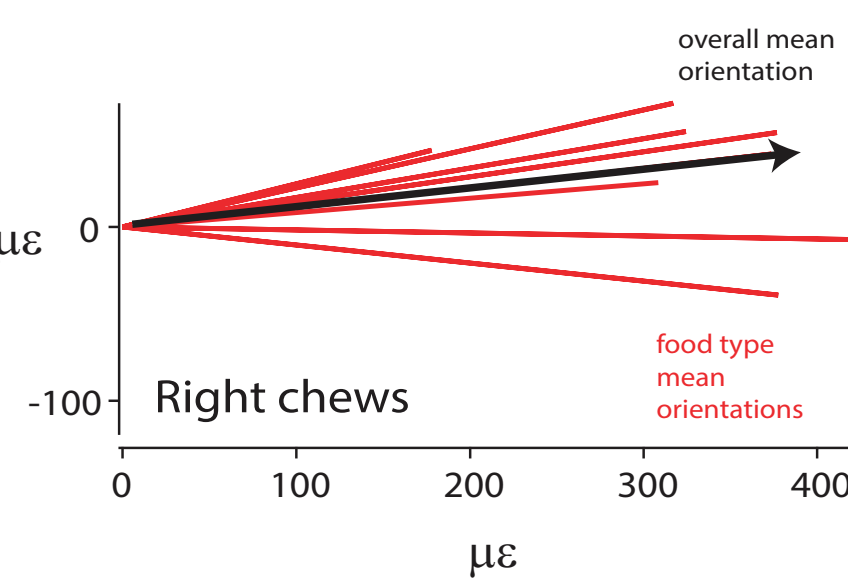


figure 9

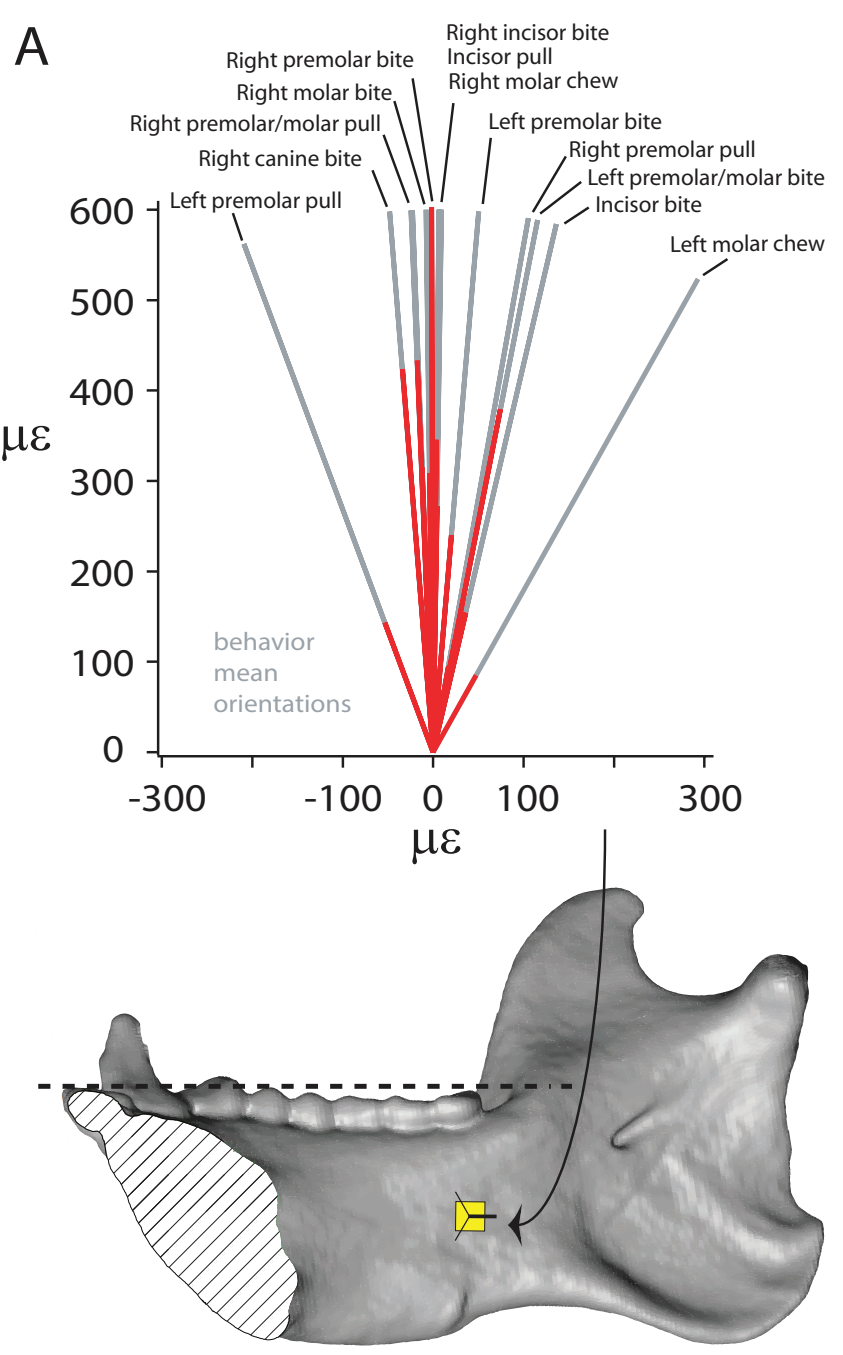

\section{Experiment 184}

Medial gage

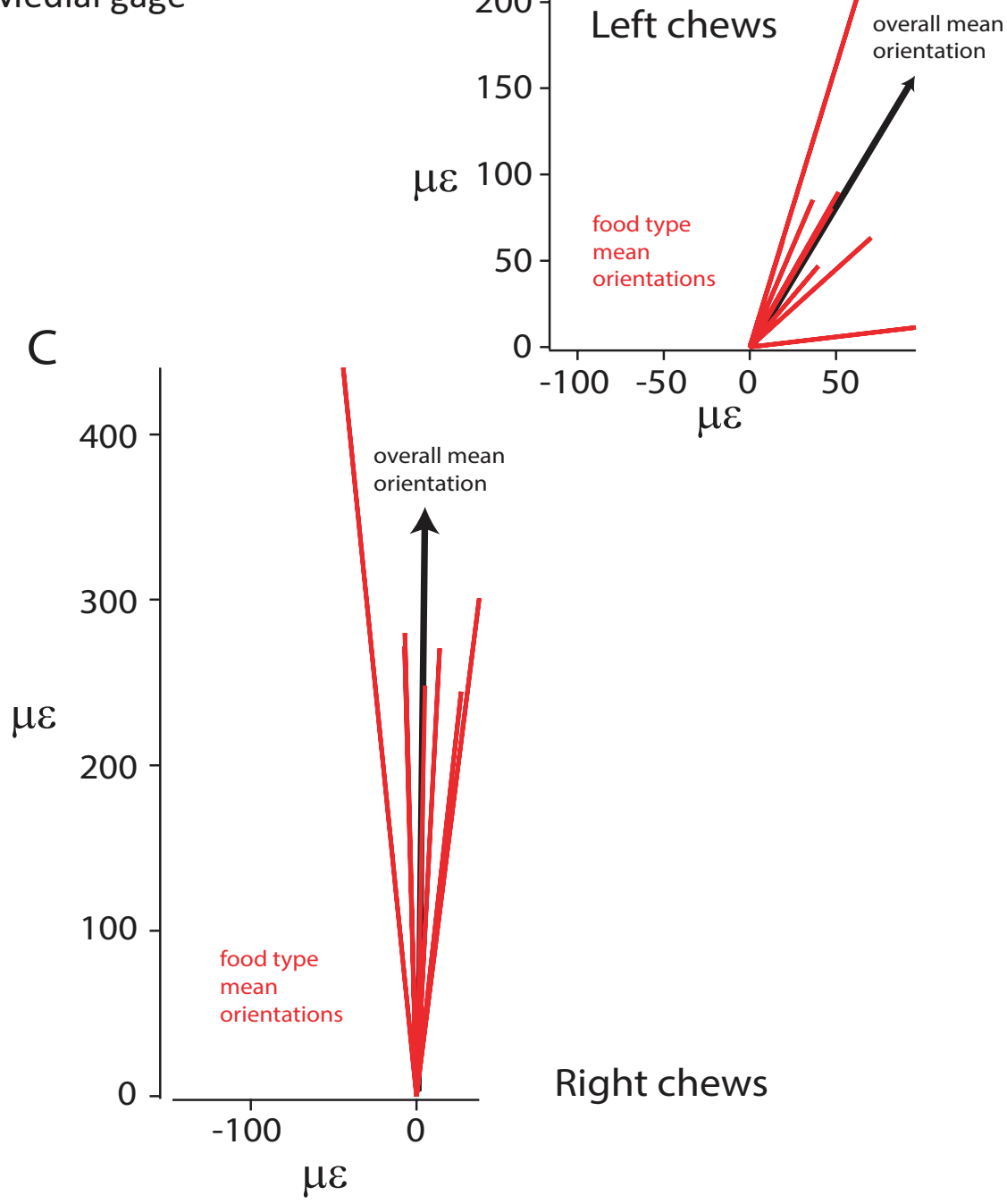




\section{figure 10}

\section{Experiment 185}

A

Upper lateral gage

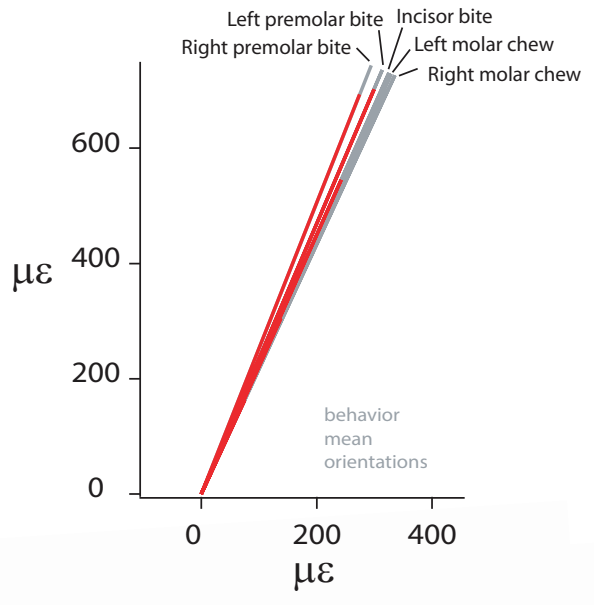

B

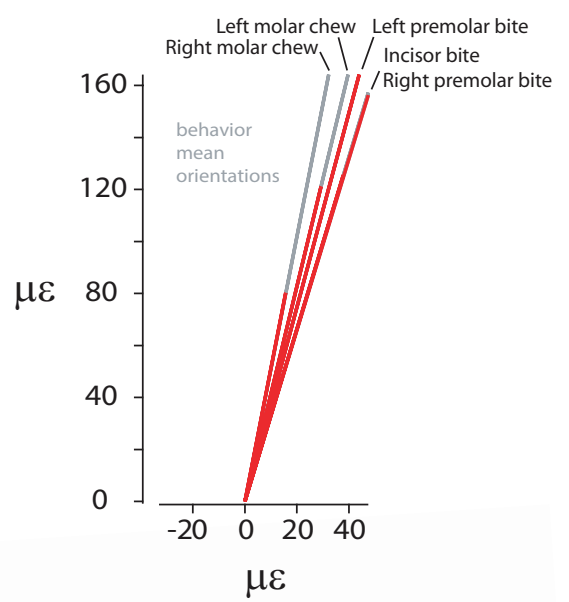

C

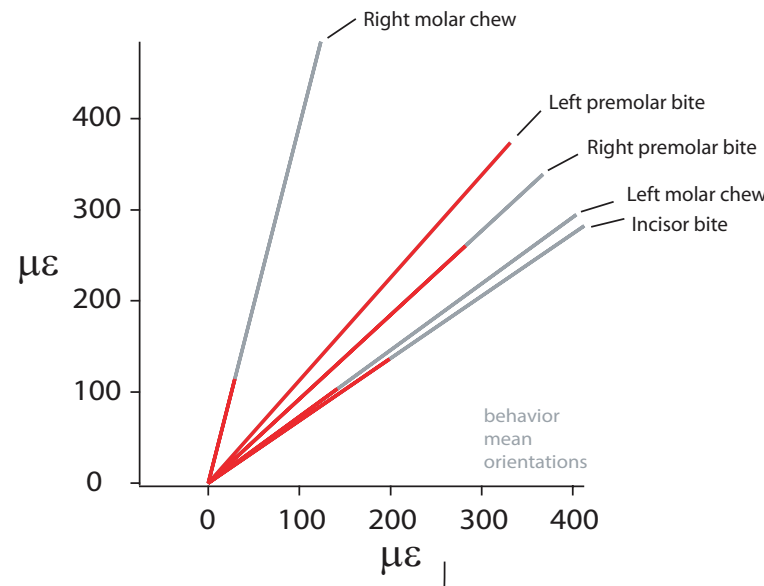

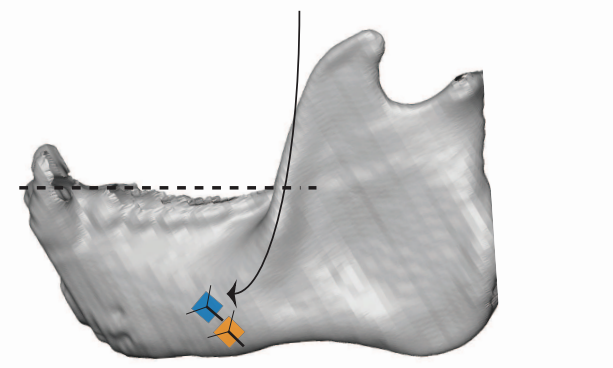
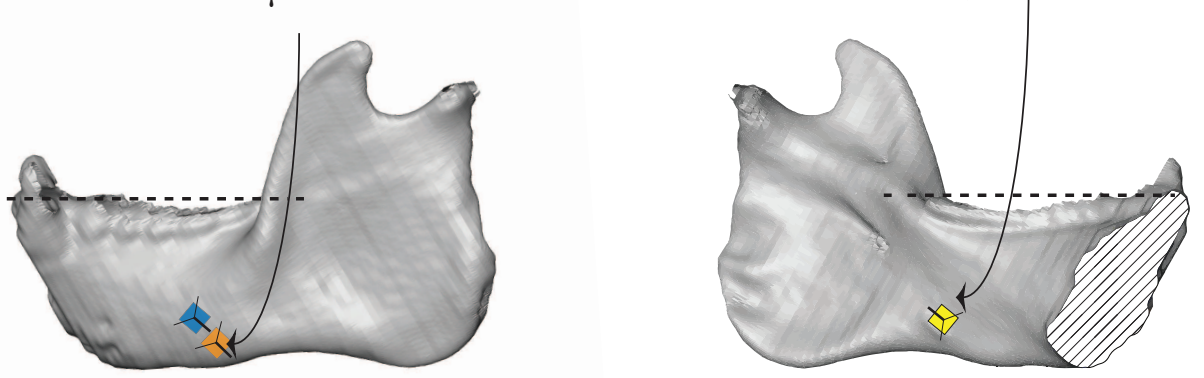
figure 11

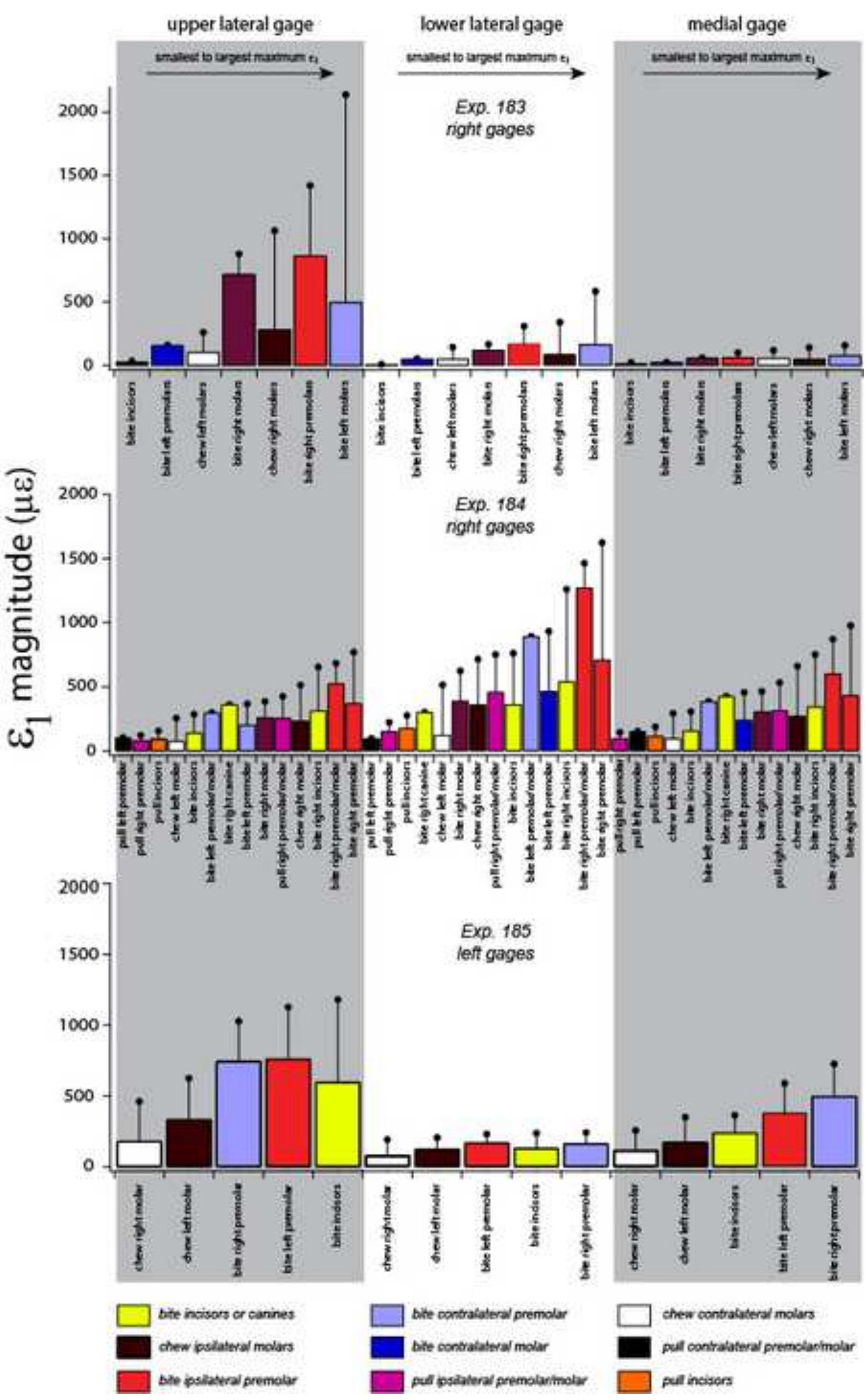


A.

\section{Food material and geometric properties}

\begin{tabular}{|c|c|c|c|c|}
\hline $\begin{array}{l}\text { Intisor } \\
\text { biting }\end{array}$ & Mastication & $\begin{array}{c}\text { Feeding } \\
\text { behaviors }\end{array}$ & $\begin{array}{l}\text { Moler } \\
\text { Biring }\end{array}$ & $\begin{array}{l}\text { Premola } \\
\text { biring }\end{array}$ \\
\hline
\end{tabular}

Joint Bite
foress forces Loading regimes $\begin{gathered}\text { Arm } \\ \text { sulling } \\ \text { forces }\end{gathered}$ Muscle

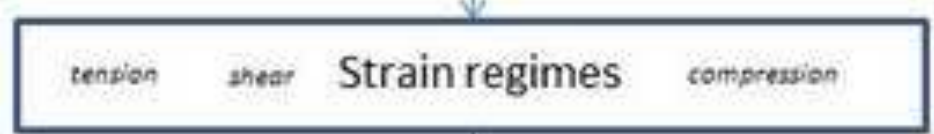

Mandible morphology
B.

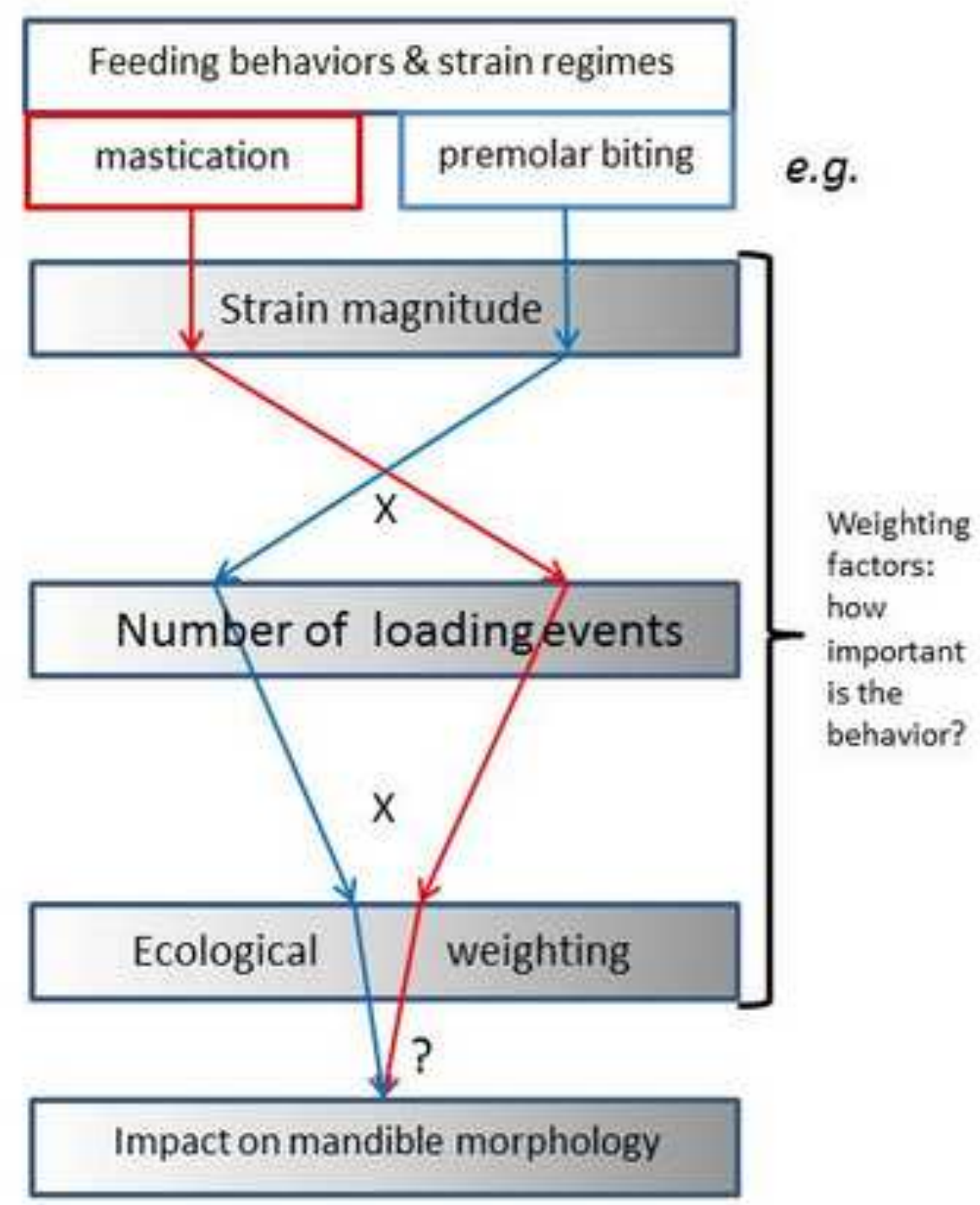

low high

